\title{
Guérir les blessures, faire naître l'espoir: Le partenariat tanzanien contre la fistule obstétricale
}

Thoraya Ahmed Obaid

Erica Chong

Population Council

Follow this and additional works at: https://knowledgecommons.popcouncil.org/departments_sbsr-pgy

Part of the International Public Health Commons, Maternal and Child Health Commons, Obstetrics and Gynecology Commons, Social and Behavioral Sciences Commons, and the Women's Health Commons How does access to this work benefit you? Let us know!

\section{Recommended Citation}

Obaid, Thoraya Ahmed and Erica Chong. 2004. "Guérir les blessures, faire naître l'espoir: Le partenariat tanzanien contre la fistule obstétricale," Quality/Calidad/Qualité no. 16. New York: Population Council. 
Guérir les blessures, faire naître l'espoir :

Le partenariat tanzanien

contre la fistule obstétricale

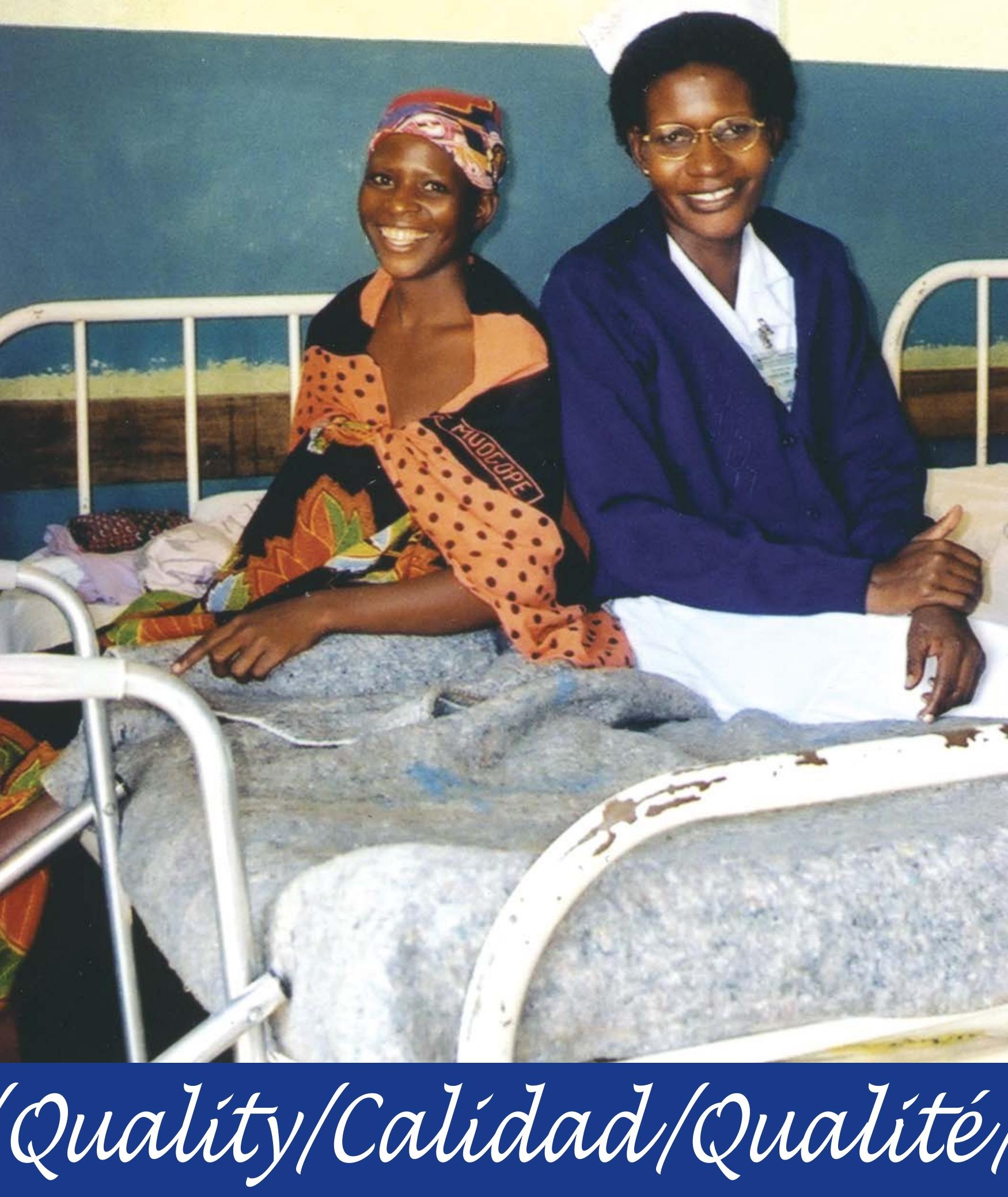




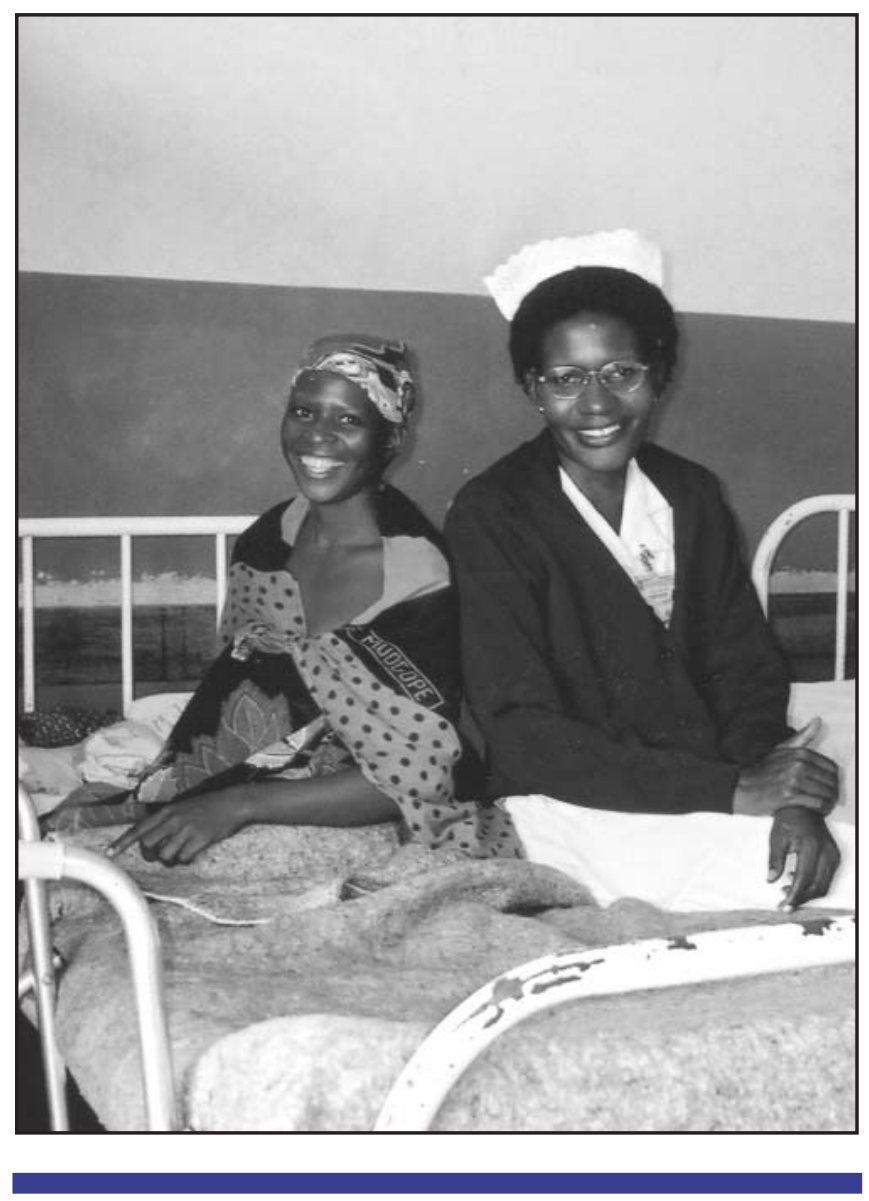

Guérir les blessures, faire naître l'espoir :

Le partenariat tanzanien contre la fistule obstétricale 
Quality/Calidad/Qualité, une publication du Population Council, met en lumière des exemples de programmes cliniques et éducatifs qui témoignent de leur engagement résolu à fournir des soins de haute qualité en santé sexuelle et reproductive et qui emploient des approches novatrices et réfléchies. La série est fondée sur la philosophie selon laquelle toute personne a fondamentalement droit à un traitement respectueux de la part des prestataires de soins de santé reproductive ainsi qu'à l'information, au choix et au suivi des soins.

$Q / C / Q$ documente des projets qui font d'importants progrès d'une ou de plusieurs des manières suivantes : en élargissant le choix de méthodes et de technologies disponibles; en fournissant l'information dont les clients ont besoin pour opérer des choix éclairés; en permettant aux clients de devenir des gardiens plus efficaces de leur santé sexuelle et reproductive; en faisant des efforts novateurs pour accroître la capacité de gestion et développer les compétences des prestataires de services à tous les niveaux; en combinant les soins de santé, la planification familiale et les services connexes de manières novatrices et en s'efforçant d'atteindre des groupes mal desservis et défavorisés.

Les projets documentés sont choisis par un groupe consultatif composé d'individus possédant une vaste expérience en matière de promotion de la qualité des soins de santé sexuelle et reproductive. Aucun de ces projets n'est offert comme un modèle à reproduire. Chacun d'entre eux est plutôt présenté comme un exemple de valeurs, d'objectifs et d'exécution révélant une créativité exceptionnelle. Ils représentent des expériences d'apprentissage qui illustrent l'attitude autocritique requise pour anticiper les besoins des clients et trouver des moyens abordables de les satisfaire. Cette attitude réfléchie se manifeste par la volonté des projets de répondre aux changements qui surviennent dans les besoins des clients ainsi qu'aux plus grandes transformations économiques et sociales qui affectent les sociétés. La documentation des choix critiques faits par ces programmes devrait aider à renforcer, en pratique, la conviction que la satisfaction de l'individu à l'égard des services de santé sexuelle et reproductive est étroitement liée à la réalisation de buts sanitaires et démographiques plus larges.

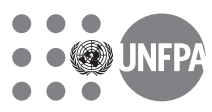

12 Population Council
Cette édition française de Quality/Calidad/Qualité a été publiée grâce au soutien de le United Nations Population Fund (UNFPA), le Robert H. Ebert Program on Critical Issues in Reproductive Health du Population Council, la Fondation Ford, ainsi que du programme « Genre, famille et développement » du Population Council. Les déclarations faites dans cette publication ainsi que les opinions exprimées sont l'entière responsabilité des auteurs et non d'une quelconque organisation soutenant $Q / C / Q$.

Le Population Council est une organisation non gouvernementale internationale, à but non lucratif, qui cherche à améliorer le bien-être et la santé reproductive des générations actuelles et futures dans le monde entier, et à favoriser un équilibre humain, équitable et durable entre personnes et ressources. L'organisme mène des recherches dans le domaine biomédical, des sciences sociales et de la santé publique, et il aide à développer les capacités de recherche dans les pays en développement. Fondé en 1952, le Population Council est dirigé par un conseil d'administration international. Son siège, qui se trouve à New York, soutient un réseau mondial de bureaux régionaux et nationaux.

Population Council, One Dag Hammarskjold Plaza, New York, New York 10017 USA

téléphone: 212-339-0500 fax: 212-755-6052 courriel:qcq@popcouncil.org http://www.popcouncil.org

Photographie de couverture : Erica Chong

Publication No 16 ISSN : 1097-8194

Copyright $(2004$ The Population Council, Inc.

Tout ou partie de cette publication peut être reproduite ou adaptée pour répondre à des besoins locaux, sans autorisation préalable du Population Council, à condition que les parties reproduites soient diffusées gratuitement ou à prix coûtant (sans profit) et que la source soit indiquée. Le Population Council serait heureux de recevoir un exemplaire de toute publication dans laquelle le texte serait utilisé. 


\title{
Introduction
}

\author{
par Thoraya Ahmed Obaid
}

Dans de nombreuses parties du monde, les femmes apprennent à accepter les risques de maladie, de blessure ou de mort, comme prix à payer pour donner naissance à un enfant. La mort d'un demi-million de femmes et de jeunes filles chaque année le prouve. Parmi celles qui survivent à I'accouchement, beaucoup voient leur santé compromise. Malheureusement, ces femmes souffrent inutilement, car la plupart des complications liées à l'accouchement sont évitables. Une si grande morbidité liée à la maternité qui persiste au XXIe siècle est le reflet de l'écart énorme qui persiste entre les pays développés et ceux en développement, en particulier dans la qualité et la disponibilité des soins de santé maternelle.

Un exemple de disparité de soins particulièrement poignant est le problème de la fistule obstétricale (voir I'encadré sur la fistule obstétricale et ses conséquences médicales). La fistule obstétricale a pratiquement disparu dans les régions riches du monde, et pourtant chaque année, l'Organisation mondiale de la santé estime à au moins 50000 à 100000 le nombre de femmes et de jeunes filles qui développent une fistule-la quasi-totalité vivant dans des régions pauvres. Ce problème semble particulièrement courant en Afrique.

\section{La pauvreté en est}

\section{la cause première}

La plupart du temps la fistule est provoquée par un travail obstrué, mais la vulnérabilité à cet état commence bien avant l'accouchement et a comme origine la pauvreté. Un retard dans le développement dû à une malnutrition peut provoquer une croissance pelvienne inadéquate et augmenter les risques de travail obstrué. De même, les filles mariées très jeunes, souvent pour des raisons économiques, se retrouvent fréquemment enceintes avant d'avoir terminé leur propre croissance. Les femmes plus âgées affaiblies par de nombreuses grossesses peuvent également être plus exposées à ce risque. De plus, ces femmes vivent très souvent dans un environnement où leur propre santé n'est pas une priorité et où leur accès aux services de soins de santé est totalement inadéquat.

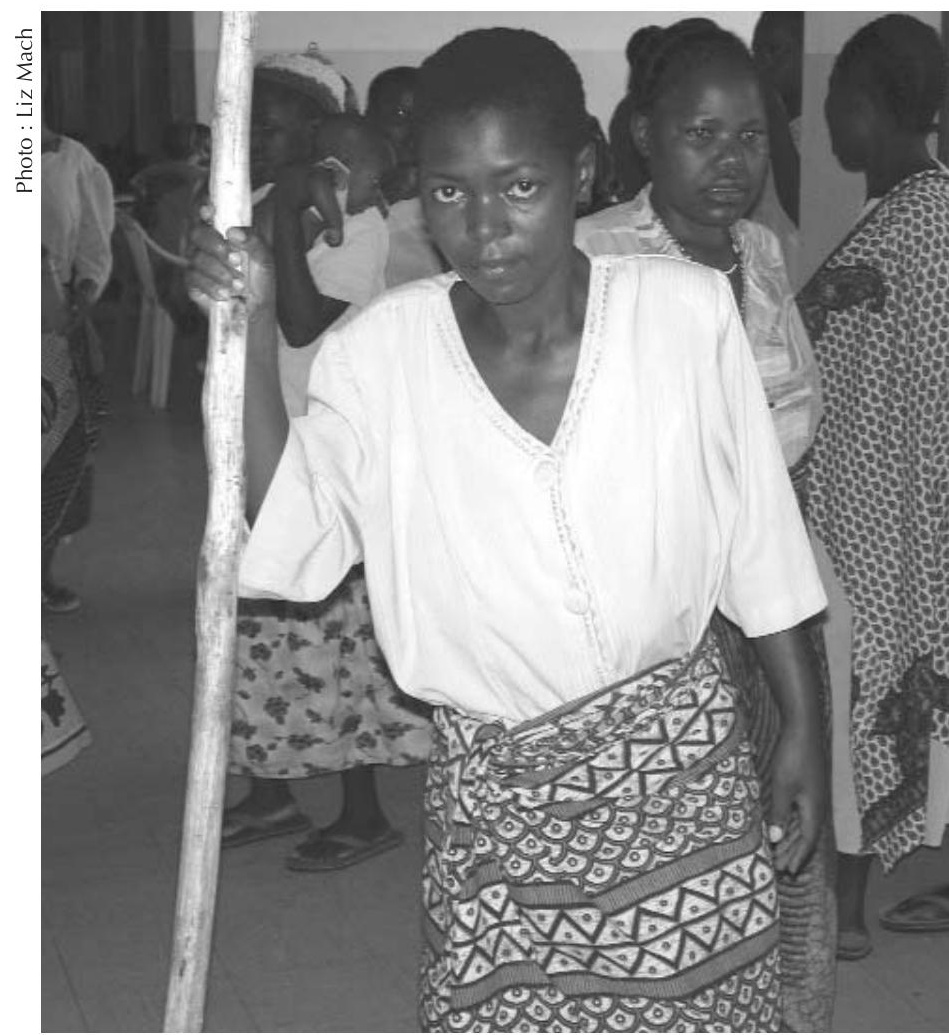

Les femmes ayant une fistule obstétricale souffrent d'un certain nombre de conséquences médicales, dont l'incontinence. Les conséquences sociales peuvent cependant être encore plus traumatisantes, avec souvent la perte des réseaux de soutien social et économique, en plus de la mort de leur bébé pendant I'accouchement. 
Les conséquences d'une fistule sont graves. Les femmes ayant une fistule souffrent $d^{\prime}$ un certain nombre de conséquences médicales, dont I'incontinence, mais les ramifications sociales sont bien plus traumatisantes. En général, une femme souffrant d'une fistule doit non seulement faire face à la mort de son bébé, mais aussi à la perte des réseaux de soutien social. Elle risque d'être rejetée par son mari parce qu'elle est devenue incontinente et sale. Il arrive

\section{Les fistules peuvent être réparées}

par une intervention chirurgicale, des mois ou même des années

après leur apparition, et une grande partie des symptômes, voire tous, peuvent ainsi être éliminés ou allégés afin que la femme puisse retrouver sa dignité et une vie normale.

même que ses propres parents refusent de I'accueillir. Elle se retrouve exclue de la vie communautaire, et elle est parfois ridiculisée, voire rendue responsable de ce qui lui est arrivé. Malgré ces épreuves, les femmes qui font face à une fistule font souvent preuve d'un courage et d'une résilience extraordinaires.

\section{Une fistule n'implique pas forcément une souffrance à vie}

Les fistules peuvent être réparées par une intervention chirurgicale, des mois ou même des années après leur apparition, et une grande partie des symptômes, voire tous, peuvent ainsi être éliminés ou allégés afin que la femme puisse retrouver sa dignité et une vie normale. Cependant, le nombre de services de réparation des fistules et de chirurgiens qualifiés est encore bien en deçà des besoins. Beaucoup de pays travaillent à l'amélioration et à l'élargissement des soins de santé, dans un contexte de concurrence féroce dû au manque de ressources (en particulier de chirurgiens). Dans de telles conditions, s'occuper d'une femme qui souffre d'une fistule depuis des années est rarement une priorité. De plus, la plupart des femmes et des jeunes filles qui souffrent d'une fistule n'ont pas connaissance de cette possibilité de réparation, et de toute façon n'ont pas les moyens de payer le traitement ou même de se rendre au centre de chirurgie.

\section{La fistule est évitable}

Pour la prévention, l'accès aux services de soins est capital; on peut sauver la vie des mères et éviter $d^{\prime}$ autres handicaps, y compris la fistule obstétrique, par la combinaison de plusieurs facteurs : des soins prénatals et des dépistages de bonne qualité, du personnel qualifié lors de l'accouchement, des traitements rapides pour les urgences, et de bons soins postnatals. Cependant, l'élimination de la fistule obstétricale est une tâche qui dépasse la sphère des services de soins. Cela exige une approche intégrée, avec le soutien et l'engagement de chaque pays individuellement et de la communauté internationale. Cela exige également un partenariat complet avec les communautés afin de promouvoir la prévention et la réparation. Des efforts d'éducation sont nécessaires pour mettre fin à l'opprobre et pour s'assurer que les familles et les communautés prennent conscience des stratégies de prévention. Une stratégie de prévention intégrée doit comprendre des initiatives qui améliorent l'équité pour les femmes en matière d'accès aux soins, d'enseignement, de revenus, et de prise de décision autonome. Des méthodes judicieuses en 
vue d'améliorer les services et de favoriser les changements sont particulièrement importantes dans les régions rurales et reculées. Les femmes qui ont bénéficié d'une réparation de fistule peuvent aussi avoir besoin d'un soutien social et de services pour les aider à retourner dans leurs communautés.

\section{La Tanzanie: une étude de cas d'efforts nationaux}

L'étude de cas suivante décrit les efforts actuels en Tanzanie pour éliminer la fistule obstétricale. Des responsables de programmes hospitaliers, d'organisations non gouvernementales (ONG), et le Gouvernement tanzanien travaillent ensemble pour recenser et traiter les femmes qui souffrent $d^{\prime}$ 'une fistule, tout en cherchant à définir et à traiter ses causes premières. L'étude fait part de I'histoire des femmes et des jeunes filles qui se rendent au Centre médical de Bugando, et celle du personnel dévoué de I'hôpital, de ceux qui plaident la cause de la communauté, et des autorités gouvernementales qui ont compris qu'il fallait soigner et prévenir la fistule et qui font tout pour répondre à ce besoin.

\section{L'UNFPA et ses partenaires-une initiative mondiale}

En 2003, le Fonds des Nations unies pour la population (UNFPA) a lancé une campagne mondiale pour mettre fin à la fistule, reconnaissant ainsi ce problème de santé féminin longtemps ignoré. En partenariat avec les organismes des Nations unies, les gouvernements, les organisations non gouvernementales, les institutions médicales et des spécialistes du secteur privé, la campagne cherche à sensibiliser, évaluer les besoins, accroître les traitements, et aborder les causes de la fistule obstétricale. Des études récentes menées au Bangladesh et au Yémen ont montré que la fistule obstétricale est un pro-

\section{La prévention et le traitement de la fistule obstétricale ne sont pas seulement un problème de pratique médicale; c'est aussi un problème de droits de la personne humaine, y compris le droit à des soins de santé adéquats en matière de procréation.}

blème que l'on rencontre aussi en dehors de l'Afrique, et les efforts de la campagne ont par conséquent été étendus à ces régions aussi. L'UNFPA et ses partenaires ont achevé l'évaluation des besoins dans 14 pays, et ils prévoient de les achever dans tous les pays à risque d'ici à 2005. Actuellement, I'UNFPA apporte son soutien à plus de 20 pays, principalement en Afrique subsaharienne et en Asie du Sud. Dans toutes les régions, I'UNFPA et ses partenaires mettent au point des stratégies nationales pour apporter des solutions aux écarts recensés en matière de prévention et de traitement de la fistule obstétricale, et apportent leur soutien à la mise en œuvre de ces plans.

La clé de l'action est de ne pas se laisser submerger par l'ampleur et la complexité du problème. Chaque intervention chirurgicale signifie la possibilité d'une vie nouvelle pour une femme. Chaque réparation réussie montre à la communauté que la fistule n'est pas une condamnation à vie et accroît la confiance dans les services de soins. Chaque sage-femme qui reçoit une formation et chaque installation qui est modernisée afin de fournir des soins obstétricaux d'urgence de qualité, contribuent à la prévention de cette condition. Chaque 
effort d'éducation dans la communauté peut sensibiliser les familles à l'importance d'assurer aux femmes les soins dont elles ont besoin.

La prévention et le traitement de la fistule obstétricale ne sont pas seulement un problème de pratique médicale; $c^{\prime}$ est aussi un problème de droits de la personne humaine, y compris le droit à des soins de santé adéquats en matière de procréation. Les femmes et les jeunes filles du monde entier méritent le temps, les ressources et l'engagement nécessaires pour atteindre ce but. Le moment est venu de remplacer une totale négligence par une action efficace, assurant à chaque femme qui met un enfant au monde de l'espoir et de la dignité.

\section{Qu'est-ce que la fistule obstétricale?}

La fistule obstétricale désigne un trou qui s'ouvre pendant le travail, en déchirant les tissus entre le vagin et la vessie ou entre le vagin et le rectum, ou les deux. En général, dans les pays en développement, la cause immédiate de la fistule est un travail obstrué dû à une disproportion céphalopelvienne (lorsque le bassin de la mère est trop étroit pour permettre le passage de la tête du bébé). Dans les services obstétricaux d'urgence adéquatement équipés-en particulier pour les césariennes-les prestataires de soins peuvent gérer la plupart des cas de travail obstrué. Quand il n'y pas d'équipement pour effectuer une césarienne ou que celle-ci n'est pas faite à temps, la pression effectuée par la tête du bébé de manière prolongée peut faire mourir et tomber les tissus du vagin, créant ainsi une fistule, ou un trou dans les tissus. Dans presque tous les cas de fistule, le travail obstrué cause également une mort néonatale.

Les femmes souffrant d'une fistule peuvent être victimes de toute une série de conséquences médicales, parmi lesquelles I'incontinence, des infections de la vessie et des ulcérations génitales. Près d'une femme sur cinq peut aussi être victime d'une baisse de pied-des dégâts aux nerfs d'une ou des deux jambes qui détériorent ainsi la capacité de marcher (UNFPA et EngenderHealth, 2003). Pour de nombreuses femmes, le cycle menstruel normal est perturbé et certaines ne peuvent plus du tout concevoir.

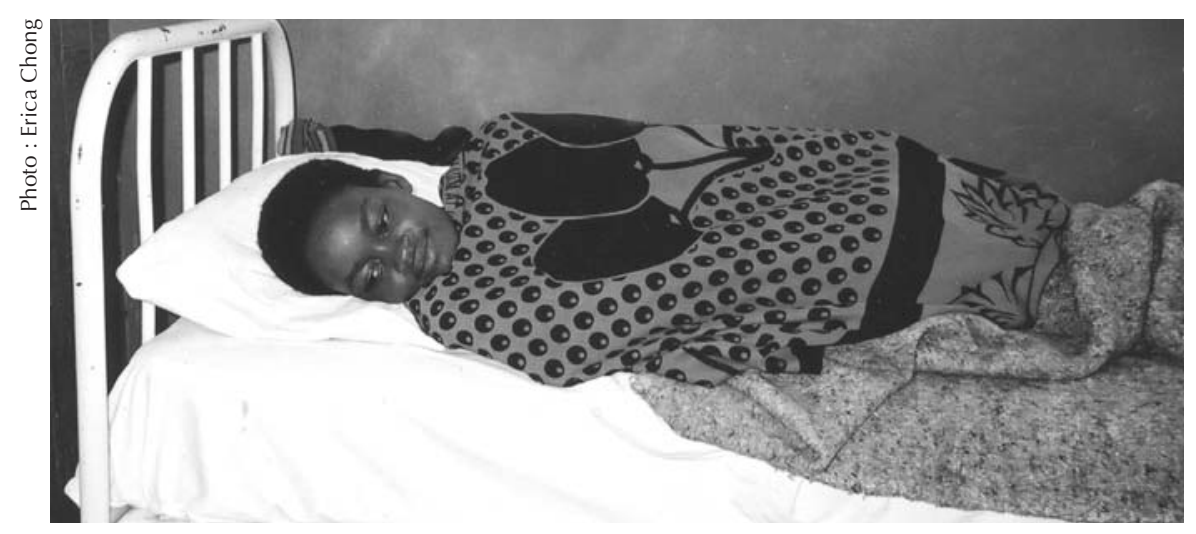

\section{Référence}

Fonds de Nations unies pour la population (UNFPA) et EngenderHealth. 2003. Rapport d'évaluation des besoins liés à la fistule obstétricale : conclusions sur neuf pays africains. New York, Organisation des Nations unies. 


\title{
Guérir les blessures, faire naître l'espoir : Le partenariat tanzanien contre la fistule obstétricale
}

\author{
par Erica Chong
}

Une fille avec une fistule rectovaginale est arrivée à l'hôpital. Quand le médecin l'a examiné, il a découvert un trou béant; l'urètre, le vagin, le rectum, tout était mélangé. Une chirurgienne invitée a travaillé pendant une heure-et-demie pour réparer la vessie et reconstruire l'urètre. Elle a fait une pause pour se détendre les épaules et s'entretenir avec les autres chirurgiens, puis a repris la procédure. Une heure-et-demie plus tard, elle avait reconstruit le rectum et le sphincter anal. Quand elle en est arrivée à la reconstruction du vagin, toutes les salles d'opération avaient entendu parler de ses talents, et quelques chirurgiens étaient venus observer la procédure. Pendant que la chirurgienne travaillait, un silence admiratif régnait, mais quand elle eut terminé, l'excitation a éclaté-certains applaudissaient, d'autres riaient, ayant du mal à croire ce dont ils avaient été témoins. Un médecin s'est mis à chanter un hymne, clair et très beau, et au fur et à mesure qu'il était rejoint par d'autres, le son est monté et a empli la salle. J'ai commencé alors à comprendre la grandeur de ce que nous pouvions accomplir, à quel point nous pouvions fondamentalement améliorer la vie de ces femmes.

— Liz Mach, Centre médical de Bugando

La Tanzanie est un pays d'Afrique de l'Est d'une beauté spectaculaire, sans doute plus connu des étrangers pour la richesse de sa faune et de sa flore dans des régions comme le Parc national de Serengeti ou le Cratère Ngorongoro. Les langues officielles sont le swahili et I'anglais, mais les 35 millions d'habitants du pays appartiennent à plus d'une centaine de groupes ethniques, chacun avec sa langue et ses coutumes.

Malgré ses richesses naturelles, la Tanzanie lutte avec les problèmes du développement; environ $60 \%$ de la population vit avec moins de 2 dollars par jour (PNUD, 2003), et I'espérance de vie est tombée à 43 ans (Banque mondiale, 2003). Les dépenses de santé du Gouvernement sont de 6 dollars par habitant, et le pays n'a que quatre médecins pour 100000 habitants (comparé par exemple à la Namibie, qui a 29 médecins pour 100000 habitants) (PNUD, 2003). Le système éducatif autrefois solide de la Tanzanie est en perte de vitesse depuis quelques années. L'abolition des frais de scolarité à l'école primaire a contribué à augmenter les inscriptions de manière significative, mais les coûts engendrés par les livres, uniformes, et autres frais divers empêchent certains enfants d'aller à l'école. Environ $5 \%$ seulement des enfants correspondant à la classe $\mathrm{d}^{\prime}$ âge suivent un enseignement secondaire (PRB, 2000).

Les filles sont souvent retirées de l'école plus tôt que les garçons, ce qui leur donne peu de compétences leur permettant de gagner leur vie, et augmente ainsi la probabilité qu'elles auront des pratiques sexuelles à risque afin de survivre économiquement. La pauvreté pousse aussi les parents à marier leurs filles tôt pour éviter le coût de les élever. L'âge moyen d'un premier mariage est de 18,3 ans en Tanzanie (PRB, 2000), mais l'âge minimum légal est de 15 ans 
pour les filles et 18 ans pour les garçons, et il n'est pas rare que les filles soient mariées à 15 ou 16 ans.

Dans une société qui juge encore la valeur des femmes à leur capacité à faire des enfants, on exerce une pression sur les filles pour qu'elles tombent enceintes le plus tôt possible après le mariage; la contraception avant d'avoir eu un premier enfant n'est pas une option envisageable. Environ $39 \%$ des filles de 18 ans sont déjà mères ou enceintes de leur premier enfant (PRB, 2001). Malheureusement, un grand nombre de ces grossesses précoces ont des issues dramatiques : les naissances et les avortements effectuées dans de mauvaises conditions causent la mort

À cause des difficultés de transport pour aller à l'hôpital, beaucoup de femmes accouchent chez elles.

Parce que son bébé ne venait pas, Rhobi a parcouru 22 kilomètres à pied et à vélo, d'où un membre du personnel de la clinique l'a conduite à l'hôpital. Quand on lui a enfin fait une césarienne, son bébé était déjà mort et Rhobi avait développé une fistule. écoulement permanent d'urine et/ou de matières fécales, et le nourrisson est généralement mort-né. Les femmes et les jeunes filles souffrant d'une fistule peuvent aussi subir des dégâts aux nerfs des jambes, rendant la marche difficile ou impossible.

La fréquence de la fistule obstétricale en Tanzanie est difficile à évaluer. Les données des hôpitaux sont très en deçà de la réalité car de nombreuses femmes ne parviennent pas jusqu'à une salle de travail d'hôpital. Les statistiques des hôpitaux sur les réparations chirurgicales indiquent un nombre encore plus réduit de patientes qui savent que la fistule peut être guérie et qui réussissent à se procurer la somme nécessaire pour I'opération. Les enquêtes sont très difficiles à effectuer à cause de l'opprobre associé à cet état, des efforts nécessaires pour atteindre les zones reculées où on trouve un grand nombre de cas de fistules, et parce que les pauvres de Tanzanie ne s'expriment pas. Néanmoins, on estime à 1200 le nombre de nouveaux cas de fistules obstétricales en Tanzanie chaque année. En 2000-2001, environ 700 femmes et jeunes filles ont subi une intervention de réparation de la fistule obstétricale, ce qui fait un retard de 500 femmes en une seule année (WDP, 2002). Si I'on multiplie ce nombre par les décennies pendant lesquelles les femmes n'ont reçu aucun traitement, le retard potentiel est immense.

\section{Causes de la fistule}

En Tanzanie, la pauvreté fournit généralement la base à des issues malheureuses en matière de santé maternelle et de procréation, et à des facteurs spécifiquement liés à la fréquence des fistules. La malnutrition et les grossesses précoces

\footnotetext{
${ }^{1}$ L'avortement est illégal en Tanzanie, sauf si la vie de la mère est en danger. Les avortements sont par conséquent effectués clandestinement, souvent avec une issue désastreuse.
} 


\section{Obstacles à l'accès aux soins}

Pour différentes raisons, les femmes retardent ou évitent le moment d'aller à l'hôpital pour accoucher. Les plus courantes sont le manque d'argent pour payer les soins et le transport, les distances à parcourir pour arriver au lieu des soins, un manque de pouvoir de décision, et l'ignorance des complications possibles pendant le travail.

Ndebele avait 19 ans quand elle a développé une fistule. ${ }^{2}$ Elle était souvent malade pendant sa grossesse et voulait accoucher à l'hôpital, mais sa belle-mère ne l'a pas laissé faire. À sept mois de grossesse, le travail a commencé et elle a accouché chez elle d'un enfant mort-né. Une fistule s'était développée pendant le très long travail, et elle a commencé à avoir une incontinence deux jours plus tard.

Rhobi avait déjà donné naissance à trois mort-nés à l'âge de 19 ans, et son dernier accouchement lui a causé une fistule. Elle vivait dans une zone rurale de l'ouest de la Tanzanie, loin de tout dispensateur de soins qualifié. Après avoir eu des difficultés à accoucher chez elle, elle a parcouru 22 kilomètres à pied et à vélo jusqu'à ce qu'elle atteigne le centre de soins le plus proche. Le personnel était incapable de l'aider, aussi l'ont-ils mise à l'arrière d'une jeep pour la conduire à l'hôpital le plus proche, distant de 25 kilomètres, où le médecin lui a fait une césarienne. Mais Rhobi était en travail depuis des jours, et son bébé était déjà mort. Elle avait développé une fistule, mais personne dans cet hôpital local ne savait la réparer, aussi est-elle restée à l'hôpital pendant plusieurs mois.

Mwasiti avait 22 ans lorsqu'elle est tombée enceinte. Son ami voulait l'épouser, mais le père de la jeune fille a refusé ce mariage. Au cours de sa grossesse, Mwasiti s'est rendu cinq fois à la clinique anténatale ; elle y a subi des examens et on lui a donné de la prophylaxie antipaludique et des comprimés de fer. Ses parents ont décidé qu'elle accoucherait à la maison parce que l'hôpital était trop cher et trop éloigné. Après un travail de trois jours, Mwasili est allée à pied à l'hôpital public, à 10 kilomètres de chez elle. Quand elle est arrivée, le médecin n'était pas disponible et les infirmières ne savaient pas comment l'aider. Quand le médecin a enfin pu la voir et lui faire une césarienne, le bébé était mort. Elle a commencé à devenir incontinente quelques jours plus tard.

Bahati avait 28 ans quand elle a accouché de son cinquième enfant. Elle avait été enceinte pour la première fois à l'âge de 21 ans, lorsqu'elle a également épousé son ami et est partie vivre chez sa belle-famille. Ses quatre autres accouchements avaient eu lieu à la maison, elle ne voyait donc pas de raison d'aller à l'hôpital pour le cinquième. $\mathrm{Au}$ cours de ses quatre visites à la clinique anténatale, elle a été vaccinée contre le tétanos et reçu des comprimés de fer. Après deux jours en travail, l'accoucheuse traditionnelle qui l'assistait lui a dit d'aller à l'hôpital. Le temps qu'elle y arrive et qu'on lui fasse un accouchement par ventouse, son bébé était mort. Bahati est devenue incontinente alors qu'elle était encore à l'hôpital, mais on ne lui a pas posé de sonde, et personne ne lui a expliqué ce qui s'était passé.

sont chacun des facteurs favorisant la disproportion céphalopelvienne. La malnutrition commence tôt et a des effets à long terme; parmi les enfants de moins de cinq ans, près d'un tiers ont un poids inférieur à la norme et près de la moitié d'entre eux accusent un retard de croissance pour leur âge (PNUD, 20003). Les grossesses précoces ne sont pas aussi courantes en Tanzanie que dans d'autres pays ayant de forts taux de fistules (par exemple l'Éthiopie et le Nigéria), mais plus d'une Tanzanienne sur quatre devient mère avant l'âge de 18 ans.

Les difficultés de transport empêchent de nombreuses femmes d'accoucher

\footnotetext{
${ }^{2}$ Les prénoms ci-dessus sont fictifs afin de protéger la vie privée des personnes concernées.
} 


\section{Vivre avec une fistule}

Les souvenirs que Ndebele garde de cette période après le travail sont flous. Elle soutient fermement que sa belle-mère est une sorcière et lui a jeté un sort. Ses parents l'ont reprise chez eux pour s'en occuper et l'ont bien traitée malgré son incontinence permanente. Ses amis et voisins sont souvent venus la voir, et certains ont pleuré de la voir dans un tel état. Ndebele est furieuse contre son mari de ne pas avoir tenu tête à sa mère quand elle était enceinte, et de ne pas être venu la voir une seule fois chez ses parents. Elle a entendu dire qu'il s'était remarié.

La mère et la sœur de Mwasiti l'ont beaucoup soutenue après son retour de l'hôpital. D'autres n'ont pas été aussi gentils : son oncle et sa belle-sœur se sont moqués d'elle, et ses voisins l'ont tenue à l'écart. Son ami l'avait soutenu pendant sa grossesse, mais a cessé tout contact avec elle quand elle a développé une fistule. Des lésions nerveuses dans l'une de ses jambes, dues au travail prolongé, lui ont provoqué une légère paralysie et lui rendent la marche difficile. Elle ne pouvait plus aider dans les champs et a cessé d'aller à l'église.

En plus de devoir supporter le chagrin d'avoir perdu son bébé, et s'adapter à la vie avec une fistule, Bahati a été harcelée par ses voisins, qui la rendaient responsable de la perte de son enfant, affirmant qu'elle l'avait tué parce qu'elle avait peur d'accoucher (comme le " prouvait " son travail interminable et son besoin d'aller à l'hôpital). Peu après son retour à la maison, son mari est tombé malade. On l'a emmené à l'hôpital, où il est mort deux semaines plus tard. Bahati est restée avec sa belle-famille, mais ils la traitaient mal. Ils lui retiraient sa nourriture et ne lui parlaient plus du tout. Après trois mois traitée ainsi, elle a pris ses enfants et est retournée vivre chez ses parents. Là, elle n'était pas humiliée. Sa propre famille a compati à ses malheurs, et d'anciens voisins sont venus lui rendre visite. Son incontinence était cependant trop importante pour lui permettre d'aller au-delà du voisinage immédiat, et elle avait totalement conscience de la mauvaise odeur due à son état. Bahati a sombré dans une profonde dépression à cause de la mort de son mari, de son enfant, et à cause de la fistule. Elle a beaucoup réfléchi sur les moyens d'améliorer son état.

dans un centre de soins. Environ $78 \%$ de la population vit en zone rurale (PNUD, 2003), et peu de familles ont accès à un véhicule ou peuvent se permettre de payer le transport. Par ailleurs, les femmes et jeunes filles ont rarement leur mot à dire sur les soins de santé qu'elles peuvent recevoir. Généralement, c'est leur mère, bellemère ou mari qui décide. Même dans des cas de travail très difficile, une femme peut perdre un temps très précieux parce qu'elle doit attendre que son mari rentre du travail et lui donne la permission d'aller chercher de l'aide ou lui donne de l'argent pour payer le voyage jusqu'à I'hôpital. Même si la majorité des femmes parviennent à obtenir des soins anténatals (le nombre moyen de visites est de quatre), il est plus compliqué de se déplacer pour aller accoucher. Les distances peuvent être plus importantes, et la date ne peut être ni anticipée ni décidée à l'avance.

Comme on I'a dit ci-dessus, la fistule a des conséquences extrêmes sur la santé des femmes et des jeunes filles. L'écoulement permanent d'urine et/ou de matières fécales produit une odeur terrible et contribue à créer des ulcérations de la peau génitale et des infections fréquentes. Certaines femmes voient aussi leur capacité à marcher fortement diminuée (baisse de pied), et $d^{\prime}$ autres ne peuvent plus avoir d'enfant. Les femmes tanzaniennes souffrant 
d'une fistule connaissent aussi les séquelles émotionnelles et économiques classiques qui les accompagnent : souffrance d'avoir perdu le bébé, honte, ridicule, isolement et, fait non rare, abandon par le mari, la famille, les amis, et les membres de la communauté. Beaucoup sont dans l'incapacité de continuer à travailler, et la plupart sont trop gênées (ou pas autorisées) pour participer à certains aspects de la vie de la communauté tels que les services et célébrations religieuses. Néanmoins, comme toutes celles qui souffrent $d^{\prime}$ une fistule dans $d^{\prime}$ autres pays, les femmes tanzaniennes font souvent preuve de ressources impressionnantes et d'une forte détermination à se soutenir ainsi que leur famille et à obtenir des soins médicaux.

Depuis des années, de nombreux professionnels de la santé en Tanzanie s'intéressaient aux patientes souffrant de fistule, mais ne savaient pas jusqu'alors comment s'attaquer à ce problème très répandu. Au milieu des années 90, une série d'événements a mis en relation plusieurs personnes qui, ensemble, se sont engagées dans un lutte contre ce problème longtemps négligé. Ils ont créé le Projet fistule au Centre médical de Bugando. Certains fondateurs du projet ont par la suite assumé de nouveaux rôles dans le secteur civil ou le gouvernement et ont continué à s'occuper du problème de la fistule. Ce numéro de Quality/Calidad/Qualité présente l'histoire du Centre médical de Bugando et son partenariat actuel avec le Women's Dignity Project (Projet pour la dignité des femmes, une organisation non gouvernementale) et avec le Ministère tanzanien de la santé pour améliorer et étendre les services de réparation de la fistule, les mesures de prévention, les efforts de plaidoyer, et la formation aux niveaux communautaire, institutionnel, national et international.

\section{Commencer à zéro}

\section{Une rencontre heureuse}

Le Docteur Balthazar Gumodoka, directeur du Projet fistule, pratique la médecine depuis 1975. Il a commencé à s'intéresser à la fistule au cours de sa formation en gynécologie/obstétrique à I'hôpital Muhimbili de Dar-es-Salaam, où I'un de ses professeurs lui a montré une réparation de fistule. Nommé dans un hôpital de la région de Mara en 1982, le Docteur Gumodoka a essayé de mettre en pratique ce qu'il avait appris à Muhumbili, mais I'hôpital ne disposait pas des installations nécessaires pour aider les patientes souffrant de fistules.

Au bout de cing ans, le Docteur Gumodoka a été muté au Centre médical de Bugando à Mwanza. Bugando dispose de 850 lits et 800 soignants, ce qui en fait le second hôpital spécialisé de Tanzanie; il dessert une zone d'environ 10 millions de personnes. Ayant là de meilleures installations, le Docteur Gumodoka a décidé de recommencer les réparations de fistule. Il opérait environ 20 patientes par an, mais comme il ne parvenait pas à moderniser ses compétences et que l'hôpital n'avait pas de programme de formation pour le personnel soignant, le taux d'échec était d'environ $50 \%$.

Pendant que le Docteur Gumodoka vivait cette frustration, une professionnelle américaine de la santé d'origine totalement différente commençait à s'intéresser au problème. Maggie Bangser travaillait en Asie pour International Women's Health Coalition lorsqu'elle a entendu parler de la fistule pour la première fois, par un collègue qui organisait des programmes de réparation de la fistule au Nigéria. Maggie est partie en Afrique de l'Est en 1992 pour diriger le portefeuille santé de la procréation de la Fondation Ford. Elle a cherché à établir des contacts avec des personnes 


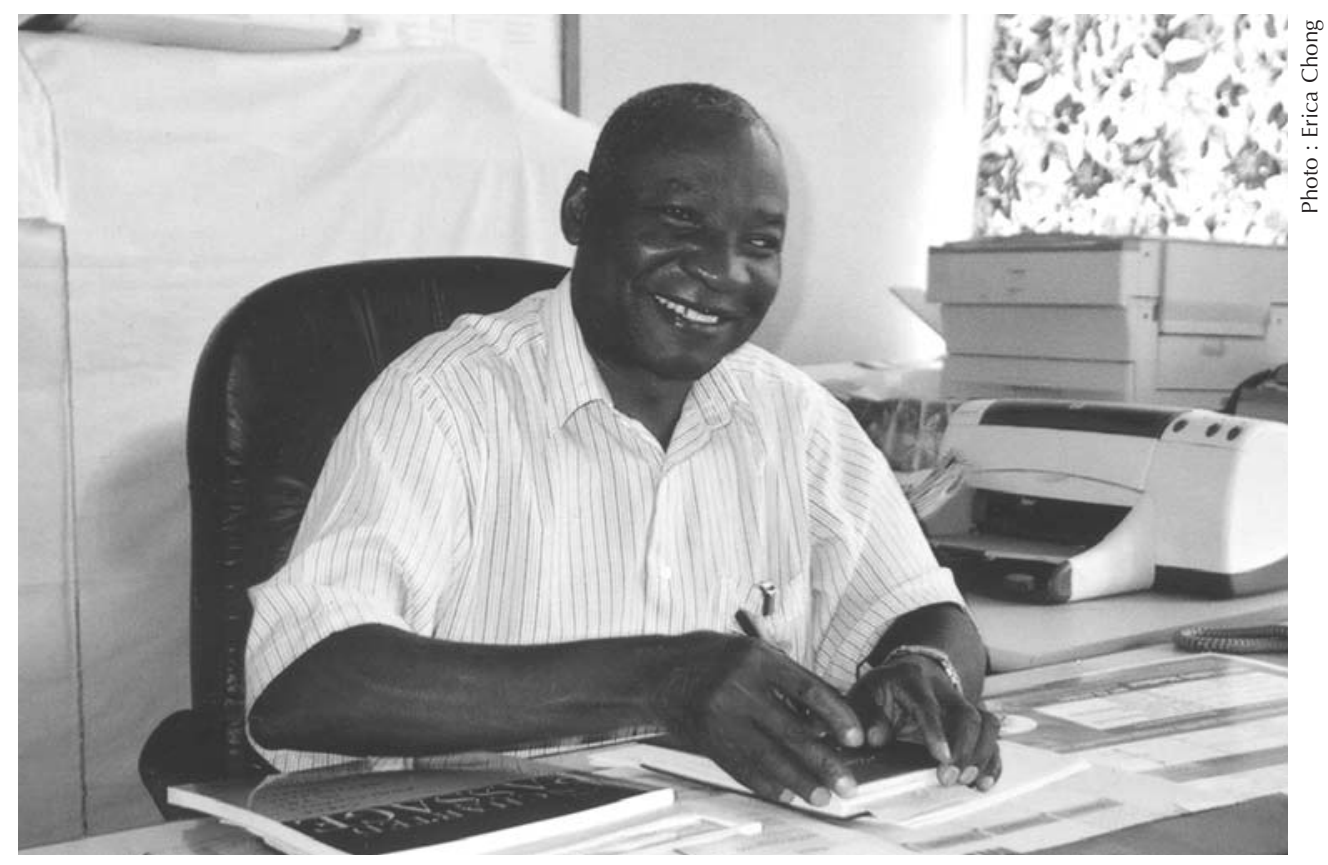

Pendant sa formation de médecin, le Docteur Balthazar Gumodoka a pu voir les effets désastreux de la fistule obstétricale. Il a commencé à s'intéresser de très près à ces patientes et à vouloir les aider, mais ses efforts ont été freinés par le manque d'installations adéquates et une formation insuffisante du personnel. Aujourd'hui, il dirige le Projet fistule au Centre médical de Bugando.

travaillant sur le problème de la fistule, mais sans succès. En 1995, elle s'est installée à Mwanza pour travailler avec une organisation non gouvernementale (ONG), où son chemin a croisé celui du Docteur Gumodoka. Au cours de la conversation, elle lui a demandé «Qu’y a-til du nouveau sur la fistule à Bugando?", et les yeux du docteur se sont éclairés. Ce jour-là, ils se sont mis ensemble à planifier tous les détails d'un vaste programme de traitement de la fistule.

Plus j'en apprenais sur l'impact de la fistule sur la vie des femmes et des jeunes filles, plus j'étais consciente que ce sujet était complètement négligé, tant du point de vue de la santé que de celui des droits de la personne humaine. La fistule est un microcosme de bien des sujets importants touchant à la vie des femmes qui vivent dans la pauvreté et des femmes qui sont marginalisées. Je me suis rendu compte qu'il était de la plus haute importance d'agir sur le problème de la fistule, mais tout aussi capital de s'occuper de tout un tas de sujets complexes qui en sont les causes, et d'apporter cette perspective pour mener à bien le travail sur la fistule.

-Maggie Bangser, membre fondatrice du Projet fistule au

Centre médical de Bugando

\section{Pose des fondements}

La première étape dans la mise au point du programme a été de créer un comité directeur, comprenant quatre autres professionnels engagés à aider les femmes et les jeunes filles souffrant de fistule et prêts à accepter des tâches supplémentaires dans leurs journées déjà bien remplies. Outre Maggie Bangser, les autres membres du comité étaient tous basés à Bugando, les médecins ou infirmières, les services de gynécologie/obstétrique, d'anesthésie ou de chirurgie. L'accent mis sur la procédure était intentionnelle 
parce que aux yeux des membres du comité, il était très important que les activités de plaidoyer et l'éducation du public n'éclipsent pas les services médicaux. Maggie se souvient : "Nous pensions qu'il était moralement contestable de sensibiliser les femmes et de leur faire espérer une réparation si les services adéquats n'étaient pas prêts ". Pendant la phase de planification, les membres du comité se réunissaient environ une fois par mois pour organiser les activités à accomplir et se partager les tâches spécifiques. Plusieurs membres du comité ont rapporté qu'une expérience personnelle auprès $d^{\prime}$ une patiente souffrant d'une fistule, et affolée, avait motivé leur participation à ce projet de traitement de la fistule.

Quand Maggie a quitté Mwanza pour créer une organisation non gouvernementale dans le but de mener des activités de plaidoyer et de recherche sur la fistule aux niveaux national et international (voir Au-delà de Bugando : un partenariat national, page 26), elle a été remplacée par Liz Mach, missionnaire laïque et infirmière Maryknoll spécialisée dans la santé maternelle et infantile, qui travaillait en Afrique depuis 1976. Liz est toujours conseillère du projet dans le comité. Quatre des six membres fondateurs du comité y sont toujours, ce qui assure une grande stabilité et continuité au projet.

Même s'il n'est pas membre du comité directeur, le Docteur Zachary Berege, directeur de I'hôpital, a été un allié précieux dans cette étape de formation. Son expérience de gynécologue-obstétricien lui avait fait voir de très près les ravages causés par la fistule. Il a apporté son soutien au nouveau projet et réservé des lits pour ouvrir un service consacré à la fistule. Lorsqu'il est devenu Directeur des services hospitaliers au Ministère de la santé, son expérience et son engagement dans ce domaine se sont révélés des atouts pour assurer le soutien suivi du gouvernement.

L'une des premières actions que le comité a entrepris a été l'élaboration des objectifs du projet, qui sont les suivants : 1) offrir des soins et des services de bonne qualité aux patientes souffrant $d^{\prime}$ une fistule qui viennent se faire soigner au Centre Bugando; 2) sensibiliser la communauté au sujet de la fistule et des manières de l'éviter, au moyen de campagnes d'information, d'éducation et de communication; 3) augmenter la capacité hospitalière de traitement de la fistule en formant des infirmières et des chirurgiens; et 4) collecter et analyser des données afin de mieux comprendre les causes et les conséquences de la fistule.

Sachant que la majorité des Tanzaniens vivent avec moins de 2 dollars par jour, une opération qui coûte entre 250 et 300 dollars dans un hôpital distant de parfois plus de 500 kilomètres est pratiquement impossible à envisager pour la plupart des femmes ou des jeunes filles qui développent une fistule. Par conséquent, vu I'importance capitale de l'opération de la fistule, les

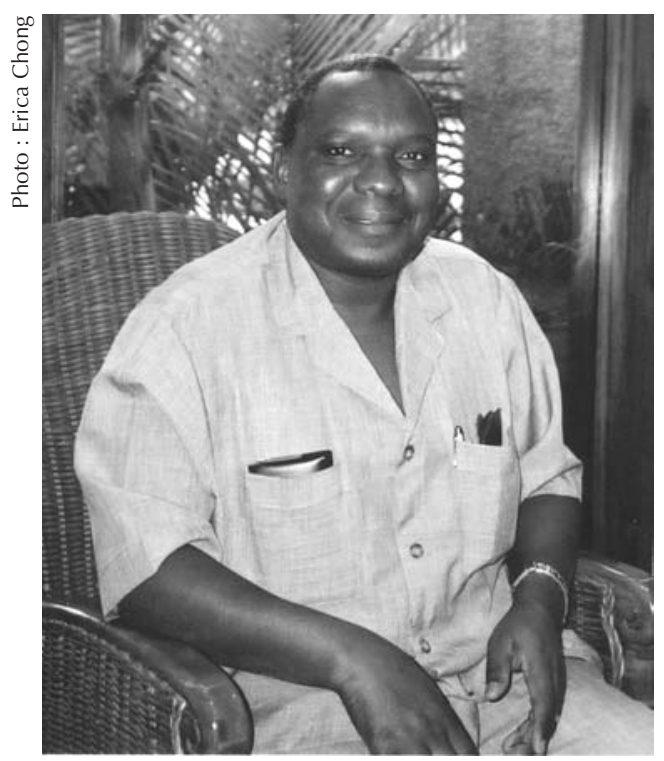

Le Docteur Zachary Berege, Directeur des Services hospitaliers au Ministère de la santé, a toujours énergiquement soutenu les services liés à la fistule. 
membres du comité sont convenus de la rendre gratuite, d'autant plus que les pauvres sont de très loin les plus touchés par ce problème.

L'un des débats menés par les personnes engagées dans le traitement de la fistule est celui concernant le lieu de traitement : est-il mieux de le pratiquer dans un lieu séparé et exclusivement consacré aux soins des patientes souf-

\section{La réparation chirurgicale de la}

fistule coûte environ 250 dollars, quand la plupart des femmes qui en ont besoin ont moins de 2 dollars par jour pour vivre. Le comité du projet a par conséquent décidé que l'opération et les soins seraient gratuits.

frant de fistules ou dans les services d'un hôpital qui existe déjà? Les partisans de la première option ont vu l'opprobre dont peuvent être victimes les patientes souffrant d'une fistule lorsqu'elles sont mêlées à d'autres patientes, et comment ce groupe mis au ban de la société est souvent repoussé en fin de chaîne des soins. Les membres du comité du projet ont été sensibles à ces arguments, mais ont aussi eu le sentiment qu'il est important de pratiquer les soins dans les services de l'hôpital afin que tout le monde puisse voir que les patientes souffrant de fistule sont comme les autres patientes, et que la fistule est un problème comme les autres, pour lequel les malades doivent se faire soigner à l'hôpital. Le comité a essayé de créer la meilleure solution à partir des deux options : il a consacré un service aux patientes souffrant de fistule dans l'aile gynécologie/ obstétrique afin, comme le dit Liz Mach, «qu'elles forment une communauté de la fistule au sein de l'hôpital, tout en faisant partie de la communauté de Bugando ». Le fait de partager les frais généraux d'électricité, d'eau et de personnel a aussi eu du poids dans la décision d'abriter le Projet fistule à Bugando.

Environ huit mois après la première rencontre entre le Docteur Gumodoka et Maggie Bangser, le plan du projet était en place; le Docteur Gumodoka est devenu le directeur du projet et Maggie la conseillère du projet. Huit mois plus tard, ils avaient obtenu les fonds et, en août 1997, I'équipe était prête à commencer la formation et à établir les services.

\section{Renforcement des capacités}

La première étape du projet a été de former le personnel médical, en commençant par les procédures de réparation de la fistule. En août, trois médecins et infirmières de I'Hôpital pour fistules d'Addis-Abeba (AAFH) (voir encadré) ont quitté I'Éthiopie pour se rendre en Tanzanie pour recevoir une formation d'une durée de trois semaines avec 55 professionnels médicaux de Bugando et d'autres hôpitaux de la région du Lac. Les spécialistes éthiopiens ont partagé leur savoir sur le dépistage, la réparation chirurgicale, les procédures en salle d'opération, les soins infirmiers pré- et post-opératoires, la gestion des cas, la mise au point de protocoles de traitement, la bonne utilisation des instruments et des solutions, ainsi que le suivi psychologique des patientes qui rentrent chez elles. Les participants ont pu observer 38 réparations de fistule pendant ces trois semaines. Sur les 38 femmes opérées, seules trois n'ont pu être guéries. Le taux de réussite de $92 \%$ était particulièrement captivant pour ceux, comme le Docteur Gumodoka, qui avaient tenté pendant des années d'aider les patientes souffrant d'une fistule mais ne disposaient ni de l'équipement nécessaire, ni de la connaissance des techniques modernes, ni des soins infirmiers adéquats.

Après cette première démonstration, le Docteur Gumodoka et une infirmière 


\section{L'Hôpital pour fistules d'Addis-Abeba (Éthiopie)}

Les docteurs Reginald et Catherine Hamlin, tous deux gynécologues-obstétriciens, ont quitté l'Australie en 1959 pour travailler en Éthiopie. Conscients des besoins énormes en réparations de fistules, ils ont créé l'Hôpital pour fistules d'Addis-Abeba (AAFH) en 1974. Depuis, l'AAFH est devenu l'un des centres chirurgicaux et de formation les plus importants d'Afrique subsaharienne. L'hôpital a déjà soigné gratuitement plus de 20000 femmes et jeunes filles et apporté aux patientes une rééducation, de la nourriture, des vêtements et de l'argent pour payer le moyen de transport pour rentrer chez elles.

L'hôpital a fait de la formation du personnel une priorité. Les gynécologues-obstétriciens éthiopiens qui n'ont pas d'expérience en matière de réparation de la fistule passent deux mois à l'AAFH dans la cadre de leur formation pratique. Les chirurgiens qui viennent d'un pays en développement et les gynécologues-obstétriciens qui ont déjà une expérience ne suivent la formation que pendant un mois. L'AAFH a récemment fait construire et ouvert Desta Mender ( « le village de la joie »), village proche de l'hôpital qui peut abriter jusqu'à 100 femmes et jeunes filles qui font de la rééducation ou dont l'opération n'a pas réussi. Ces dernières ont des sacs pour urostomie qui exigent une attention médicale régulière dont elles ne bénéficient pas dans leurs villages éloignés de tout. À Desta Mender, les jeunes femmes travaillent les champs, apprennent à tricoter et filent la soie.

qualifiée en gynécologie/obstétrique sont partis chacun à son tour suivre une formation intensive de quatre semaines en Éthiopie à I'AAFH. Redynamisés, ils sont rentrés à Bugando et ont organisé des ateliers pour faire partager à leurs collègues des services de gynécologie/ obstétrique, d'anesthésie et de chirurgie ce qu'ils avaient appris. Sur la base de ces ateliers, le projet de Bugando a adapté les protocoles AAFH à son environnement et à sa clientèle, par exemple, en introduisant de nouveaux systèmes de gestion des cas et de tenue des dossiers, en mettant à jour les protocoles de nettoyage des lits et des sondes, et en renforçant les capacités d'aide psychologique. Les systèmes de gestion financière établis par le comité du projet comprenaient un système transparent de comptabilité, ainsi que des comptes en monnaie locale et en devises étrangères, une caisse pour les dépenses courantes, et un fonds pour les besoins vitaux des patientes. Avec les subventions qu'ils avaient obtenues, ils ont acheté un équipement complet d'instruments chirurgicaux adaptés aux procédures de la fistule, trois lits et dix matelas.

Le Comité du Projet fistule du Centre médical de Bugando (BMC) collabore avec deux chirurgiens étrangers qui ont des compétences en réparation des fistules et viennent régulièrement au BMC. Le Docteur John Kelly, un médecin anglais d'environ 70 ans, répare des fistules depuis plus de 25 ans dans plusieurs pays d'Afrique et d'Asie. Le Docteur Tom Raassen, un chirurgien néerlandais, travaille pour la Fondation pour la médecine et la recherche en Afrique (AMREF) et dirige deux projets obstétricaux de réparation de la fistule en Afrique orientale. Près de deux mois après le retour de l'équipe de Bugando de I'AAFH, le Docteur Kelly est venu à Bugando pour diriger un atelier de deux semaines. Trente-cing femmes ont été soignées au cours de son passage, et les chirurgiens, infirmières et personnels de salle d'opération ont pu mettre à jour et renforcer leurs savoirs.

Il n'a pas fallu très longtemps à l'équipe de Bugando pour devenir capable d'établir un véritable programme de traitement de la fistule. Pendant les 18 premiers mois, le projet a permis de procurer à 150 jeunes femmes un traitement, de la nourriture, du savon et de I'argent pour payer un moyen de transport pour rentrer chez elles. Celles qui vivaient dans la plus 

ger est aussi souvent un supplice pour qui est incontinent. Les contrôleurs n'acceptent qu'avec réticence les femmes souffrant de fistule dans les trains ou dans les autobus. Ils peuvent même les jeter dehors si elles sentent trop mauvais où que les autres passagers se plaignent. Certaines femmes ont passé des mois, voire des années à négocier la permission d'aller se faire soigner et à économiser l'argent nécessaire pour le voyage et I'intervention chirurgicale.

Bahati souffrait d'une fistule depuis deux ans lorsqu'elle a décidé de retourner à I'hôpital où elle avait accouché pour voir si on pouvait faire quelque chose pour elle. Elle est restée dans le service une semaine et demie, avant que le médecin ne l'envoie au Centre de Bugando. N'ayant jamais quitté son village, elle avait peur de voyager seule et d'aller si loin. Le médecin lui a dit de ne pas s'inquiéter et lui a trouvé quelqu'un pour l'accompagner. Il a payé pour elle et une infirmière le voyage en bus jusqu'à Mwanza. En arrivant à Bugando, Bahati a été très surprise de constater qu'il y avait autant de femmes qui souffraient du même problème qu'elle. Elle n'avait jamais entendu parler de fistule auparavant et pensait que son cas était unique. Cela lui a donné du courage de voir qu'elle n'était pas toute seule et à partir de ce moment elle a attendu l'intervention chirurgicale avec impatience. Avant I'opération, Bahati a du être traitée pour une anémie et une infection vaginale.

\section{L'intégration des patientes souffrant de fistule dans la communauté de l'hôpital}

L'approche fondamentale du projet se démarque radicalement de la démarche hospitalière traditionnelle en accordant, à côté de la prise en charge médicale, une place prépondérante à la prise en charge sociale. Comme le souligne Liz Mach, la conseillère actuelle du projet, " après le supplice que ces femmes ont enduré, la stigmatisation et l'abandon, il est vraiment important qu'elles se sentent les bienvenues ici, qu'elles considèrent l'hôpital comme un refuge dont les portes leur sont toujours ouvertes, où elles se sentent en sécurité et traitées avec dignité ». Lorsque le projet a commencé, les gens demandaient au personnel pourquoi il mettait des draps sur les lits des patientes souffrant de fistule puisque, de toute façon, il faudrait les changer le lendemain. Mais comme dit une infirmière " ça n'a pas d'importance si les draps sont sales! Nous les lavons. Mettre des draps sur les lits c'est normaliser la situation de ces femmes et leur rendre un peu de leur dignité ».

Les infirmières, en plus de dispenser les soins médicaux, fournissent un soutien psychologique aux patientes et les aident à vivre leur convalescence. Conscientes que ces femmes ont tout perdu - enfant, mari, capacité reproduc-

Certaines femmes passent des années à négocier la permission d'être soignées et à économiser la somme nécessaire pour le voyage. Mais ensuite, le voyage peut être un supplice pour une femme qui est incontinente. Il arrive que des conducteurs de bus ou de train la chassent si elle sent trop mauvais ou si d'autres passagers se plaignent.

tive, source de revenu, amour propre, statut social—elles savent qu'elles ont besoin de temps pour faire le deuil et en parler. Elles les incitent également à réfléchir sur leur séjour à I'hôpital et leur avenir. Elles les aident à définir leurs 


\section{Espoir et détermination}

Ces femmes et jeunes filles, qu'elles arrivent des environs ou de plus loin, en général souffrent de malnutrition et ne possèdent que les vêtements qu'elles ont sur elles. En plus, comme elles boivent le moins possible pour limiter leur incontinence, beaucoup souffrent d'une déshydratation chronique provoquant parfois des calculs rénaux. Une patiente avait un calcul dans la vessie de la taille d'une balle de golfe. La déshydratation provoque une concentration d'ammoniaque dans l'urine souvent à l'origine de dermatite ou d'excoriation de la vulve. L'une des premières étapes lors de l'admission est un entretien pour déterminer si la patiente souffre d'une fistule, d'une incontinence d'effort grave ou d'une infection devant être traitée avant l'opération. Si la fistule est nouvelle (une semaine environ) on essaye d'abord de la traiter sans intervention chirurgicale (par insertion d'une sonde pour quatre à six semaines et une bonne hydratation). Environ $10 \%$ des cas peuvent être soignés de cette façon.

Ndebele souffrait d'une fistule depuis neuf ans lorsqu'elle a entendu à la radio qu'un traitement était possible. Cela lui a donné un nouvel espoir mais il lui a fallu deux ans pour rassembler l'argent nécessaire pour aller à l'hôpital. Sa famille ne possédait pratiquement plus rien après la maladie et la mort de sa mère en 2000. L'année dernière, son oncle l'a emmenée à la ville voisine où ils sont restés cinq mois pour essayer de trouver l'argent nécessaire à son voyage à l'hôpital, mais il a fallu couper court à leurs espoirs lorsque la sœur de Ndebele est décédée. Finalement, avec l'argent gagné en vendant des haricots et des cacahuètes et les sommes prêtées par des parents elle a pu aller jusqu'à l'hôpital le plus proche où les médecins lui ont mis une sonde pendant 14 jours. Mais elle n'a pas été guérie. Pendant qu'elle était à l'hôpital, Ndebele a entendu parler du Projet du Centre de Bugando et du fait que le traitement là-bas était gratuit. Après avoir tant peiné pour atteindre le premier hôpital, elle ne voyait pas comment elle pourrait aller jusqu'à Mwanza. Mais une infirmière compatissante, notant sa détresse, est intervenue auprès de l'Administration de l'hôpital et a rassemblé les fonds nécessaires à son voyage.

Mwasiti souffrait d'une fistule depuis un an lorsqu'elle a décidé de chercher de l'aide. Elle s'est rendue à Bunda à l'hôpital de la mission où les médecins ont essayé de la guérir. Mais l'intervention a échoué et Mwasiti est revenue encore plus découragée qu'avant. Dans les années qui ont suivi sa sœur l'a toujours soutenue, lui répétant qu'un jour elles trouveraient une solution et qu'elle serait guérie. Un jour elles ont entendu à la radio une émission sur la fistule obstétricale et appris que le Centre médical de Bugando pratiquait des réparations chirurgicales gratuites. Mwasiti avait peur d'essayer à nouveau. Elle ne pensait pas qu'elle pourrait jamais guérir parce que la première tentative avait échoué et elle ne voulait pas être à nouveau cruellement désappointée. Sa sœur l'a encouragée à essayer et peu après toutes les deux se sont rendues à Mwanza.

Le médecin qui s'occupait de Rhobi, après avoir entendu à la radio que le Centre de Bugando traitait les fistules obstétricales, l'a conduite à Mwanza, à deux heures de voiture sur une mauvaise piste pleine de nids de poule. Rhobi est arrivée au Centre en s'appuyant sur un bâton avec seulement un drap pour se couvrir et un morceau de plastique pour s'asseoir. Elle était couverte de gale, avait une infection respiratoire et était morte de peur parce que si loin de chez elle. Quand les infirmières lui ont expliqué la procédure chirurgicale, la vue de la seringue pour l'anesthésie péridurale l'a tellement effrayée qu'elle a presque refusé d'être opérée. Les infirmières ont passé des heures à la calmer et ont finalement réussi à la convaincre en priant avec elle, en lui demandant d'avoir la foi et en l'assurant que Dieu prendrait soin d'elle.

Hidaya, 42 ans, souffrait d'une fistule depuis l'âge de 19 ans. Son accouchement avait duré quatre jours et le bébé était mort-né. Immédiatement après l'accouchement elle avait commencé à souffrir d'incontinence et développé une lésion. Son mari l'a abandonnée à cause de sa fistule. Deux tentatives de traitement, en 1980 et 1988, ont échoué. En 2001, un travailleur sanitaire lui a parlé du projet et lui a suggéré d'essayer encore une fois. Lors de l'entretien d'admission à Bugando, on a découvert qu'elle souffrait de six fistules. Le chirurgien qui l'a opérée n'était pas optimiste. 


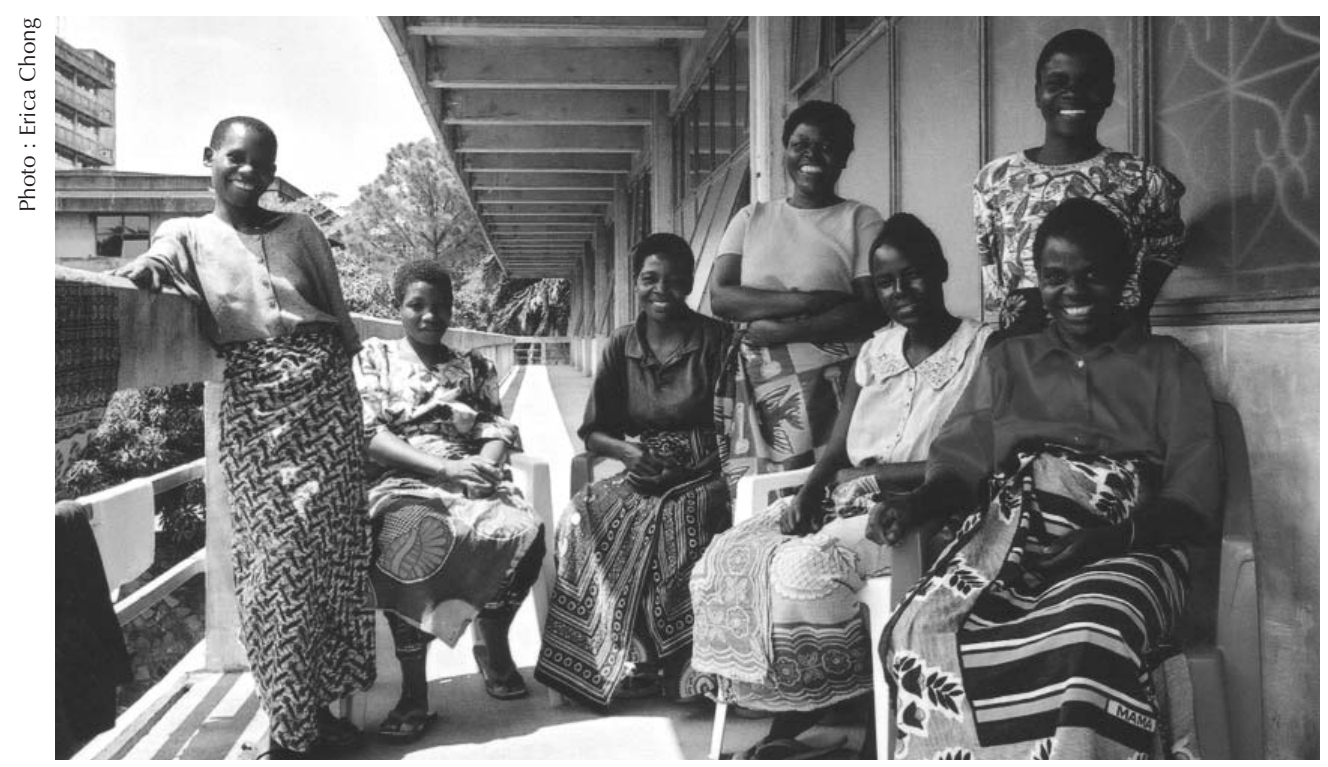

Les patientes considèrent le service des fistules comme un refuge. Pour beaucoup d'entre elles, c'est la première fois depuis l'apparition de leur fistule qu'elles se sentent acceptées et traitées avec dignité. Le fait de pouvoir discuter ensemble de la perte d'un enfant ou de l'abandon d'un mari les aide beaucoup.

besoins, leurs problèmes, leurs espoirs et leurs attentes. Certaines patientes (particulièrement les plus jeunes) s'attachent aux infirmières qui deviennent des mères de remplacement ou des grandes sœurs. Pour humaniser les soins et diminuer la peur, I'anesthésiste rencontre systématiquement chaque patiente avant I'intervention chirurgicale pour qu'elle puisse reconnaître un visage familier dans la salle d'opération.

Peut-être que l'un des aspects les plus importants de l'expérience vécue par les patientes à Bugando est le temps passé avec les autres femmes traitées dans le service. La plupart de ces femmes et jeunes filles sont surprises de découvrir qu'elles ne sont pas les seules dans leur cas. Celles qui sont arrivées à I'hôpital tristes, anxieuses et sans grand espoir de guérison sont rassurées en voyant des femmes qui ont été opérées et sont maintenant en train de se rétablir. Pour beaucoup d'entre elles, $c^{\prime}$ est la première fois depuis I'apparition de leur fistule qu'elles se sentent acceptées. Le fait de pouvoir discuter ensemble de la perte d'un enfant ou de
I'abandon d'un mari les aide beaucoup. Comme le dit Furaha, une patiente, " $C^{\prime}$ est fantastique d'être avec d'autres femmes qui comprennent ce que j'ai subi. Nous parlons entre nous de nos problèmes et de ce que nous avons vécu après l'apparition de la fistule ». Une autre patiente parle de sa colère: "Quand j'ai vu dans le service toutes ces femmes qui souffraient aussi de fistules j'ai réalisé que c'était vraiment un énorme problème. Mais pourquoi personne ne nous en a parlé avant? Si j'avais été au courant, j'aurais peut-être pu éviter ma fistule ou au moins je serais venue ici plus tôt ».

Les patientes restent dans le service de quelques semaines à plusieurs mois, cela dépend de plusieurs facteurs-le besoin d'une physiothérapie, la nécessité d'un traitement pour soigner des infections ou autres maladies avant l'intervention, le nombre de patientes à traiter, le succès de l'opération. Durant leur séjour à I'hôpital, les patientes peuvent, si les infirmières ont le temps ou s'il y a des volontaires pour leur enseigner, apprendre à parler Swahili, à tricoter, à faire du crochet ou à lire et 


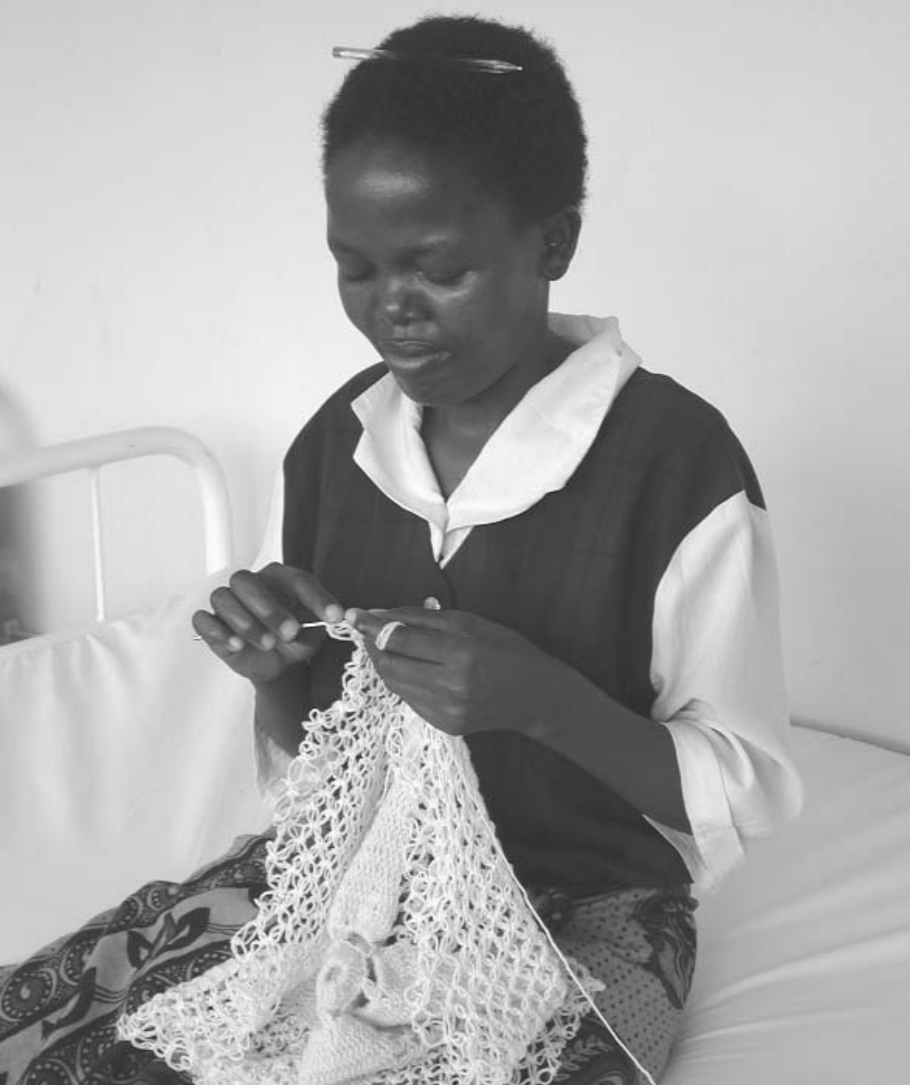

Les patientes restent dans le service de quelques semaines à plusieurs mois. Nombre d'entre elles apprennent à parler swahili, à faire du crochet ou à lire et écrire, des activités qui peuvent se pratiquer assise. Après avoir lancé un appel dans Vogue Knitting International pour recevoir des fournitures, le projet a reçu des centaines de kilos de laine et d'aiguilles à tricoter.

écrire. Liz Mach souligne que " non seulement ce type d'activité permet aux patientes de s'occuper, d'oublier leurs problèmes et d'acquérir un savoir-faire utile pour plus tard mais aussi crée des liens entre elles». Le crochet est une activité qui peut se pratiquer assise, ce qui est très important pour celles qui souffrent d'incontinence d'effort ou ne contrôlent pas encore bien leur vessie après I'opération. Un appel lancé pour recevoir des fournitures, y compris le numéro d'automne 2000 de Vogue Knitting International, a reçu une réponse fantastique. Les lecteurs ont envoyé par le biais d'AmeriCares des centaines de kilos de laine et d'aiguilles à tricoter. Tricoter permet aussi aux patientes du service de se sentir intégrées dans la communauté du Centre médical de Bugando car la grande majorité des couvertures, des pull-overs et des chaussons pour bébé qu'elles tricotent est distribuée aux malades de l'hôpital qui en ont besoin.

\section{L'opération et les soins post-opératoires}

Le nombre de réparations chirurgicales de fistules à effectuer étant très important, le projet est continuellement confronté à un problème de personnel. Le Docteur Gumodoka pratique les réparations chirurgicales simples et complexes mais il est très pris par ses responsabilités de Directeur adjoint de l'hôpital, de Chef du Département d'obstétrique/ gynécologie et de Directeur du projet. La moitié des réparations est encore pratiquée par des chirurgiens en visite. Le Docteur Kelly passe, chaque année, six semaines à Bugando pendant lesquelles il pratique environ 120 réparations; le Docteur Raassen, au cours de ses séjours d'une à deux semaines à I'hôpital, pratique environ 20 réparations.

Dans un même temps, un corps de chirurgiens locaux se constitue lentement dans le cadre du projet. Deux chirurgiens tanzaniens, le Docteur Marietta Mahendeka et le Docteur Nhandi $\mathrm{Ng}^{\prime}$ walida viennent de terminer leur formation à l'hôpital spécialisé d'Addis-Abeba.

Le Docteur $\mathrm{Ng}^{\prime}$ walida explique que sa décision remonte à l'époque où il était étudiant et faisait son stage pratique à I'hôpital. II voyait des patientes souffrant de fistule rester pendant des mois, voire des années, sans que personne ne puisse les traiter. Elles venaient le supplier d'atténuer leurs souffrances et il était désespéré de ne rien pouvoir faire. Le médecin insiste sur les abus de pouvoir qui sont à l'origine de ce fléau : " C'est parce que ce sont les parents qui décident que les jeunes filles se marient plus tôt qu'elles ne le voudraient et ont des enfants avant 
$d^{\prime}$ y être prêtes. Et quand elles sont mariées c'est la même chose, elles n'ont aucun pouvoir. C'est le mari qui contrôle l'argent du ménage et l'épouse a besoin de son autorisation pour recevoir des soins médicaux ».

La réparation chirurgicale d'une fistule peut prendre d'une à sept heures. Les opérations simples sont réalisées avec anesthésie péridurale et consistent à couper la cicatrice et à suturer. Parfois une greffe de tissu adipeux est nécessaire pour augmenter I'irrigation sanguine de la partie recousue. Les interventions plus compliquées nécessitent une anesthésie générale et peuvent inclure une réimplantation de l'uretère dans la vessie ou un conduit iléal (dérivation du trajet habituel de l'urine par un segment de l'intestin grêle vers une poche collectrice placée à l'extérieur de I'abdomen après ablation de la vessie).
Après I'opération, la patiente reste alitée pendant deux à 14 jours (cela dépend du chirurgien; la période de convalescence à I'hôpital fait encore I'objet de discussions et d'études). Bien que I'intervention soit évidemment extrêmement importante, la qualité des soins infirmiers est également cruciale, particulièrement dans la période postopératoire. Souvent, sans une bonne prise en charge médicale post-opératoire, la blessure risque de se rouvrir. Comme la patiente opérée utilise encore une sonde urinaire et que sa vessie est rétrécie (sa vessie n'a pas été utilisée depuis l'apparition de la fistule), toute distension de la vessie pour retenir l'urine peut provoquer la réouverture de la fistule. Les infirmières doivent non seulement bien prendre soin de la blessure mais également contrôler le drainage par sonde pour éviter tout risque de blocage et veiller à ce

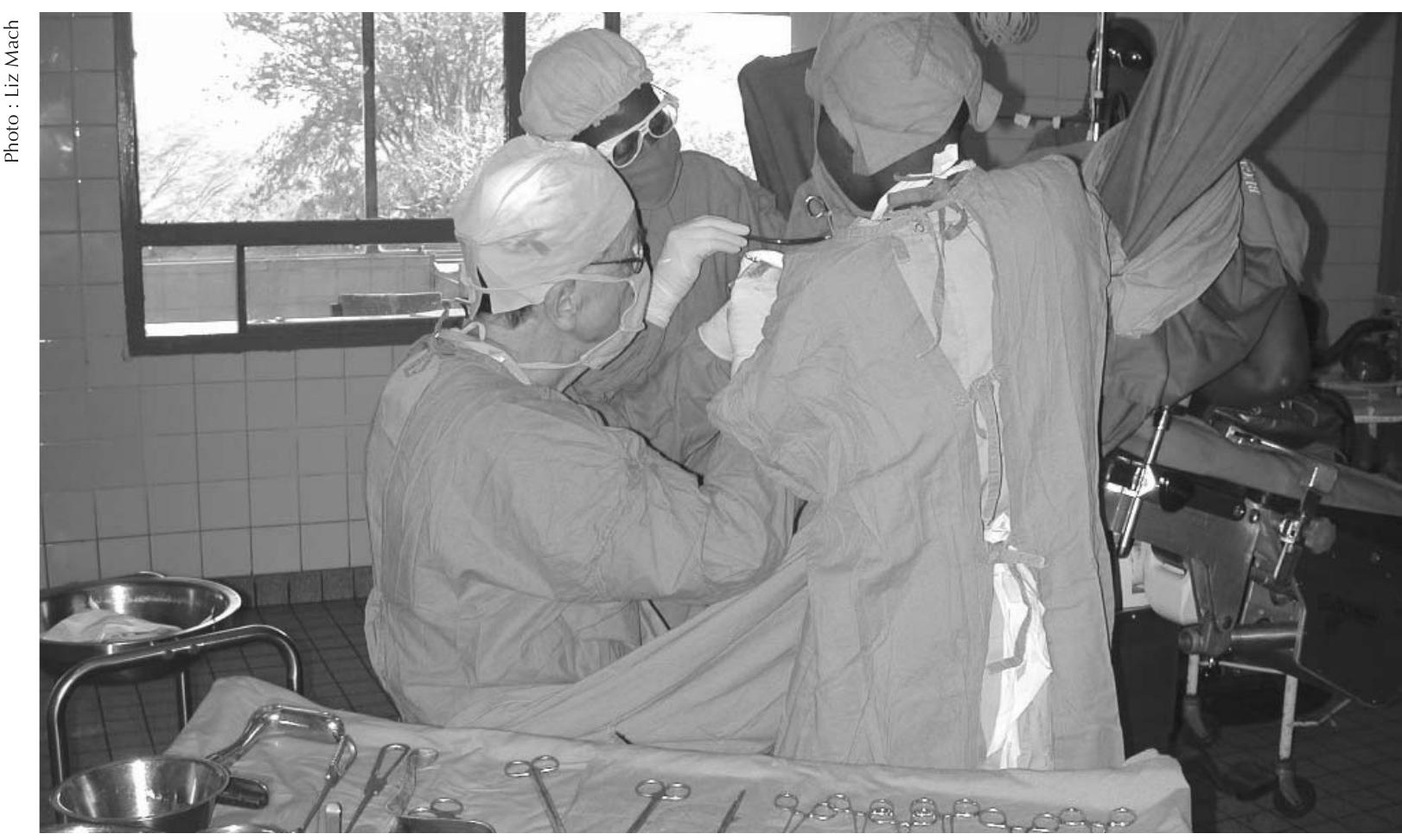

La réparation chirurgicale d'une fistule peut prendre d'une à sept heures. Un corps de chirurgiens locaux se constitue lentement mais la moitié des réparations est encore pratiquée par des chirurgiens en visite. Le Docteur Kelly (en haut) passe, chaque année, six semaines au Centre médical de Bugando. 


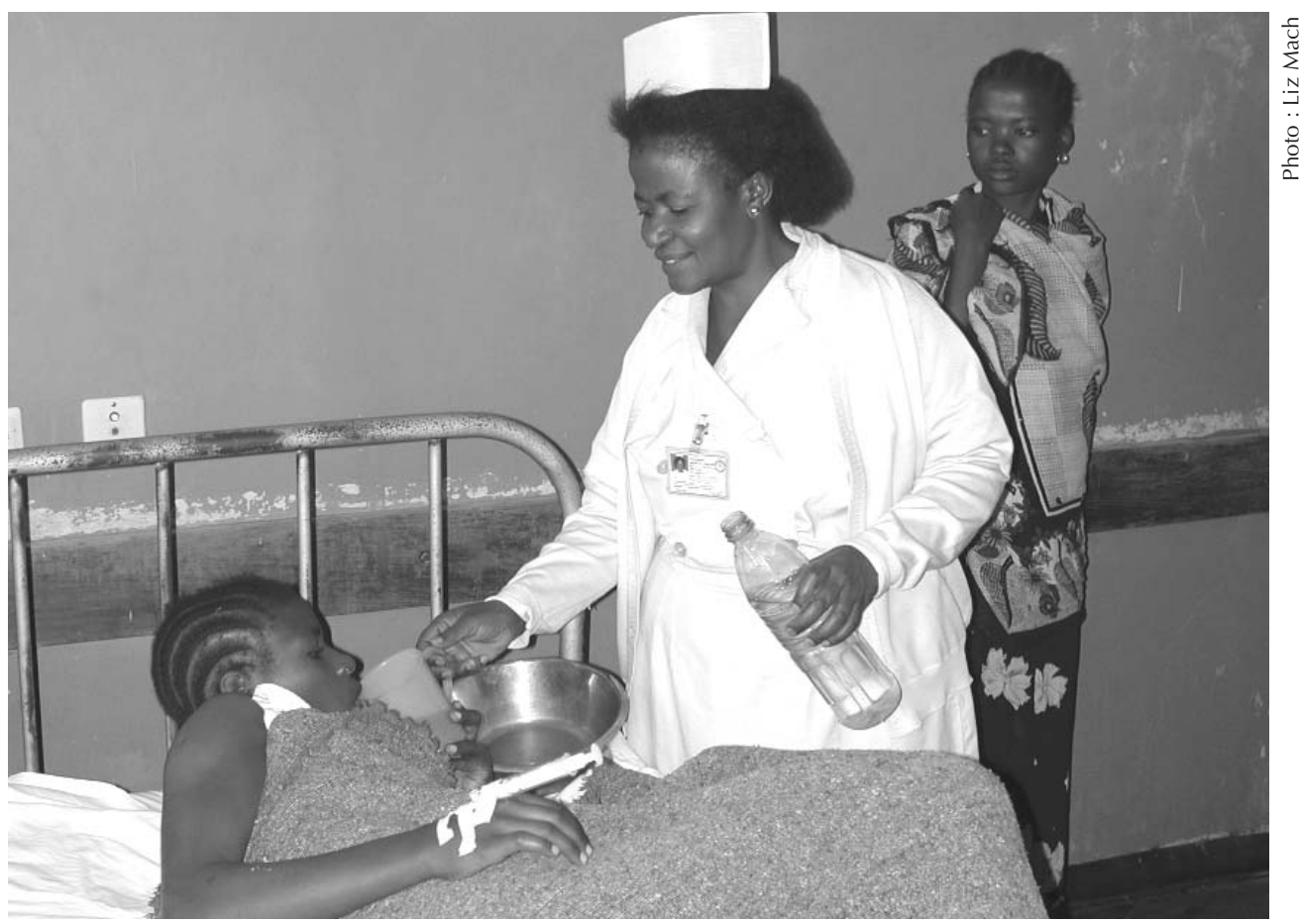

Après l'opération, la patiente reste alitée pendant deux à 14 jours. La qualité des soins infirmiers est cruciale pour la réussite de l'intervention.

que les patientes boivent suffisamment et s'alimentent correctement durant leur convalescence.

Après I'opération, certaines patientes continuent à souffrir $\mathrm{d}^{\prime}$ incontinence $\mathrm{d}^{\prime} \mathrm{ef}-$ fort, lorsqu'elles éternuent, soulèvent des objets lourds ou au réveil. D'après les infirmières la gymnastique des muscles pelviens aide, dans de nombreux cas, à retrouver le contrôle des sphincters. Liz Mach rappelle l'entretien de sortie d'une patiente qui, lorsqu'on lui a demandé si elle souffrait encore d'incontinence, a répondu « Oui j'ai encore des problèmes mais là juste maintenant je fais mes exercices, pas vous? "Cette patiente envisageait très calmement son incontinence parce qu'elle sentait qu'elle pouvait la contrôler. II est bien évident que les patientes sont soulagées lorsque leur opération chirurgicale a réussi et qu'elles n'ont plus d'écoulement d'urine ou de matières fécales. Au cours d'un autre entretien de sortie, la patiente à qui I'on demandait si elle souffrait encore d'in- continence a sauté sur ses pieds et relevé sa jupe en disant : "Regardez, je porte une petite culotte pour la première fois depuis des années! »

\section{Les résultats de l'inter- vention chirurgicale}

À Bugando on considère les femmes comme étant guéries quand elles ne souffrent plus d'incontinence. Sur 645 opérations pratiquées, $74 \%$ ont réussi, $5 \%$ ont échoué et $20 \%$ ont partiellement réussi (les femmes opérées souffrant encore, plus ou moins, d'incontinence). L'incontinence d'effort peut aller d'un écoulement bénin à un épanchement aussi important qu'avant la réparation de la fistule. Bien que dans certains cas I'incontinence d'effort puisse être atténuée grâce à une gymnastique pelvienne, dans d'autres cas, une nouvelle intervention chirurgicale est nécessaire. Parfois même la lésion est incurable. Certains cas se traitent plus difficilement 
que d'autres, notamment lorsque la patiente a déjà subi une intervention qui a échoué, le plus souvent dans un hôpital plus petit et mal équipé. Comme la zone touchée est relativement petite et que des tissus cicatriciels se forment à chaque opération, c'est toujours la première intervention qui a le plus de chances de guérison. D’après Liz «au début, on recevait beaucoup de patientes ayant déjà été opérées sans succès ailleurs et c'était très difficile de les soigner ». Parfois il est difficile de savoir pourquoi une opération échoue. Peut-être que la déchirure était très large, le tissu cicatriciel très important ou le système immunitaire de la patiente affaibli par le VIH. En cas d'échec le personnel médical prend particulièrement soin de la patiente, en l'épaulant moralement, en lui expliquant que le médecin fera une autre tentative et qu'elle ne sera pas abandonnée.

\section{Et alors comment s'est terminée l'histoire de Ndebele, Mwasiti, Bahati et Hidaya?}

L'opération de Ndebele a échoué mais elle espère que la prochaine tentative mettra enfin un terme à ses années de souffrance et de honte.

Mwasiti, à sa grande surprise, 14 jours après son opération ne salissait plus son lit.

L'opération a permis de réparer la fistule de Bahati mais la jeune femme souffre encore d'une incontinence d'effort modérée.

Et Hidaya? Après une opération réussie et plus aucune incontinence, le personnel de l'hôpital a dû l'obliger à quitter l'hôpital parce qu'elle montait et descendait les corridors en chantant, en dansant et en dérangeant tout le monde.

\section{Le retour chez soi}

Chaque patiente a un entretien de sortie avant de quitter I'hôpital. Un des objectifs de cet entretien est de compléter, à des fins statistiques, les renseignements rassemblés à son entrée, principalement les indicateurs sociaux, économiques et de santé-âge au mariage, âge à la première grossesse, parité, niveau d'éducation, soins reçus durant la grossesse et à l'accouchement, précisions sur la fistule et sur les conditions de vie. Lors de cet entretien la patiente reçoit aussi les derniers conseils pour prendre soin de sa santé et est incitée à réfléchir à la vie qu'elle va mener une fois de retour au village.

\section{Lorsqu'on a demandé à la patiente si elle souffrait encore d'incontinence, elle a sauté sur ses pieds et relevé sa jupe en disant : " Regardez, je porte une petite culotte pour la première fois depuis des années!"}

Certaines patientes n'ont jamais, au cours de leur maladie, arrêté d'avoir leurs règles, d'autres ont vu leurs règles réapparaître après l'intervention et peuvent à nouveau avoir des enfants. Les patientes reçoivent toute une série de conseils-ne pas avoir de relations sexuelles avant six mois (en disant six le personnel espère une abstinence $d^{\prime} a u$ moins trois mois), se faire suivre médicalement en cas de grossesse, accoucher à l'hôpital, informer les médecins qu'elles ont eu une fistule. Elles sont également averties qu'elles risquent de devoir accoucher par césarienne. Les infirmières cherchent à s'assurer que la patiente a pensé à son avenir-où elle va habiter, comment elle va survivre, si elle souhaite se remarier ou, si son mari est encore au village, comment est-ce qu'il va réagir à son retour? 
Quatorze jours après son opération,

Rhobi ne souffrait plus d'incontinence et était prête à rentrer chez elle. Comme elle avait été amenée à l'hôpital en voiture par le médecin du centre de santé local et qu'elle n'avait jamais quitté son village auparavant, elle ne savait pas où il était ni comment y retourner. Des membres de l'équipe ont décidé de l'aider à rentrer. Rhobi se rappelait vaguement la direction du centre de santé dont le médecin l'avait amenée à l'hôpital. Le personnel du centre a indiqué à Rhobi et à ses accompagnateurs où était son village. Mais après avoir traversé le village deux fois en voiture Rhobi n'avait toujours rien reconnu. Finalement, elle a vu un homme qu'elle connaissait sur la route qui les a emmenés à la case de la grand-mère avec laquelle elle vivait. Lorsque Rhobi est descendue de la voiture au milieu des villageois rassemblés sa grand-mère est sortie en larmes en disant "Je te croyais morte".

Certaines patientes n'ont vraiment rien qui les attende chez elles. Parfois, elles restent travailler comme aides dans le service jusqu'à ce qu'elles ont économisé assez pour pouvoir commencer quelque chose en rentrant au village. Ces anciennes patientes servent d'intermédiaires entre les patientes et le personnel hospitalier et à ce titre ont un rôle essentiel. Cette stratégie est aussi appliquée dans certains programmes d'autres pays. Le cas d'une patiente traitée à l'AAFH lorsqu'elle était adolescente est exceptionnel. Incapable de rentrer chez elle, elle est restée dans le service et a commencé à participer timidement, sous la supervision du Docteur Reginald Hamlin, aux réparations chirurgicales de fistule. Elle s'est avérée douée pour ce genre de travail et est finalement devenue elle-même chirurgienne. Elle a déjà pratiqué des milliers d'interventions et est l'une des meilleures formatrices de l'hôpital.

On sait peu de chose sur la réintégration des patientes dans leur communauté d'origine. L'un des objectifs du Projet de Bugando maintenant est de suivre les patientes après leur retour au village pour consigner leur expérience et la façon dont elles sont acceptées par leur communauté et leur mari. Est-ce qu'elles retombent dans la situation d'extrême pauvreté et d'oppression du sexe féminin qui ont généré leur problème de fistule? Est-ce qu'elles seront capables d'avoir encore des enfants si elles en veulent? Comment se passe leur grossesse? Quels sont les éléments que le projet doit prendre plus soigneusement en compte pour faciliter la réintégration des anciennes patientes? Est-ce qu'il faut plus insister sur la formation à des activités créatrices de revenu? L'équipe étudie actuellement comment

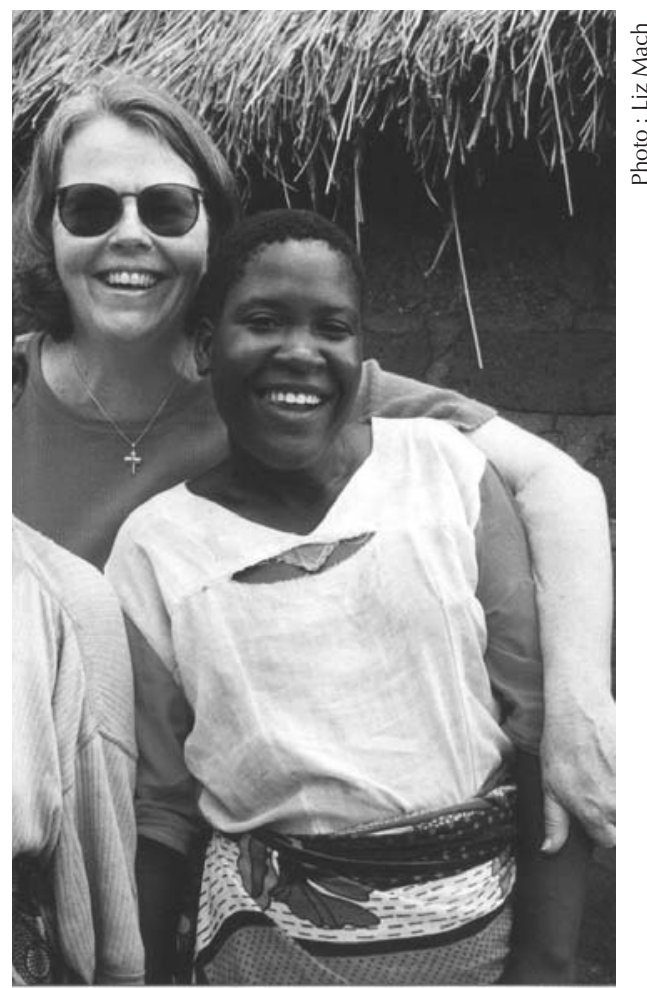

Liz Mach avec une patiente qui est rentrée chez elle. 


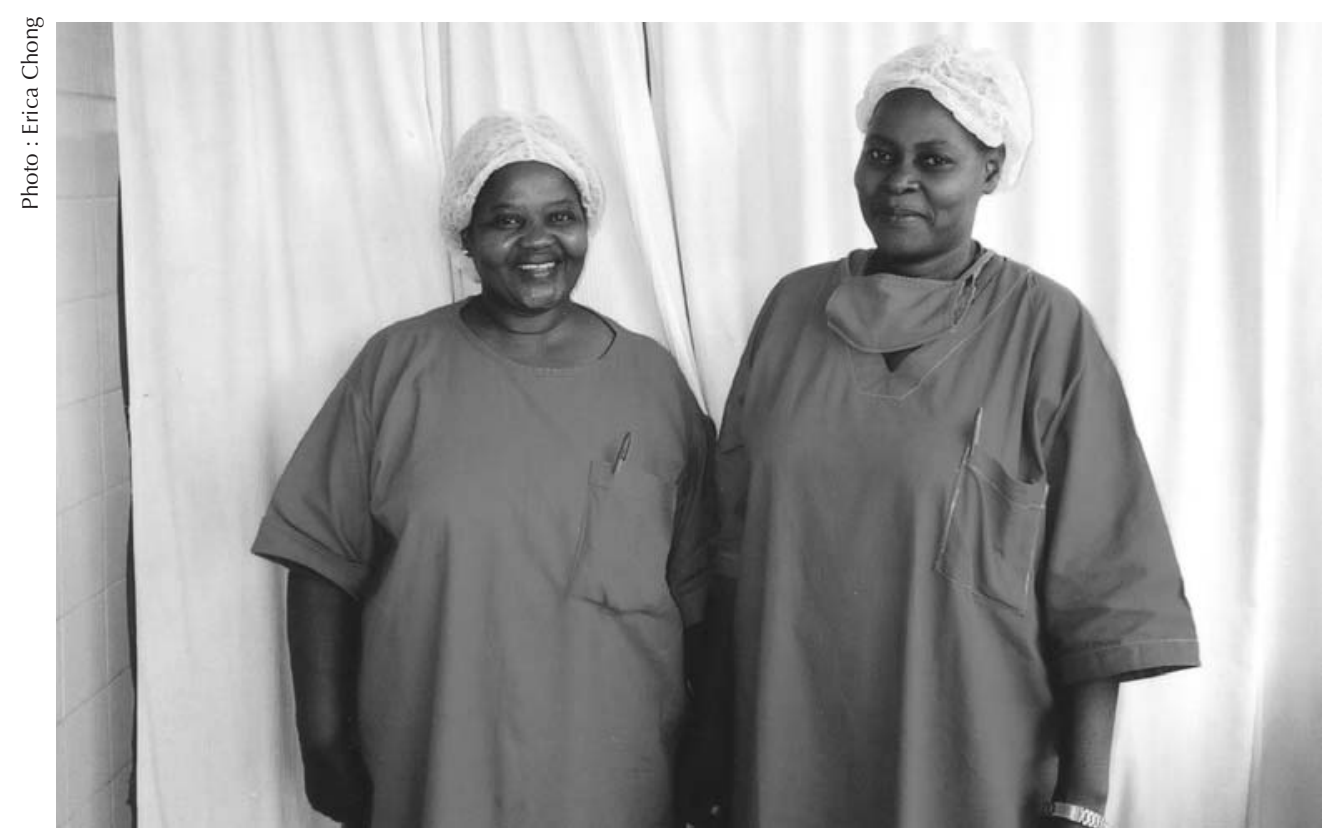

Le travail au service des fistules est difficile et effectué à titre volontaire. Néanmoins, le projet attire des infirmières dévouées et les rotations de personnel sont rares.

localiser les anciennes patientes dispersées dans le pays et rendre les entretiens plus informatifs.

\section{L'équipe du projet}

L'équipe du projet se réunit deux à trois fois par an au grand complet avec les médecins, les anesthésistes, le personnel du bloc opératoire et de l'administration. Ces réunions, qui durent en général plusieurs heures, permettent $d^{\prime}$ introduire les nouveaux membres de l'équipe, de discuter les problèmes, de préparer ou d'évaluer la visite d'un médecin étranger. Chaque participant, indépendamment de sa place dans la hiérarchie de l'hôpital, est encouragé à donner des idées pour augmenter l'efficacité du service et à parler des problèmes qu'il rencontre dans son travail. Plusieurs membres de l'équipe trouvent que ces réunions, menées dans un réel esprit d'ouverture, sont extrêmement motivantes et leur donnent vraiment le sentiment de contribuer à la réussite du projet.

\section{Les difficultés du projet}

En 2001, environ 50 hôpitaux tanzaniens pratiquaient des réparations de fistules mais six en assuraient la majorité. Le Centre médical de Bugando monopolisait environ un quart des réparations, soit plus que tout autre hôpital (WDP, 2002). Le nombre de réparations chirurgicales de fistules est passé de moins de 30 par an (lorsque le projet a commencé) à 228 en 2002, avec une chance de réussite également beaucoup plus importante. Le développement rapide du projet a amené le Comité à demander des locaux supplémentaires. En 2002, le successeur du Docteur Berege à la tête de I'hôpital, le Docteur Charles Majinge, un spécialiste des réparations de fistules, a attribué 50 lits supplémentaires au projet qui en a maintenant 95. La nouvelle section accueille les patientes attendant d'être opérées et parfois aussi des convalescentes lorsque la section principale est pleine. 
patiente traitée par un spécialiste de I'AMREF en visite dans le service. Le Centre médical de Bugando fournit les infrastructures (électricité, eau, locaux, lits, etc.) mais ne participe pas aux dépenses de traitement. Liz assure qu'aucune patiente n'a jamais été refusée par manque d'argent mais reconnaît que la lutte pour couvrir les dépenses est quotidienne et que le manque de fonds rend difficile la réalisation de certains objectifs de recherche et de diffusion.

\section{Le développement des capacités locales}

Le nombre croissant de patientes a amené le directeur de I'hôpital, le Docteur Charles Majinge, un spécialiste des réparations de fistules, à augmenter le nombre de lits de 50 à 95.

Cette augmentation de la capacité du service permet de traiter davantage de femmes mais en même temps a exacerbé certains problèmes, notamment les difficultés de personnel et de financement.

\section{Le financement du projet}

L'équipe estime que chaque patiente, compte tenu des coûts de l'intervention chirurgicale, des examens de laboratoire, des médicaments, de la nourriture, des transports pour le retour au village et des salaires du personnel, coûte environ 250 dollars. C'est grâce à Liz Mach qui s'emploie activement à trouver les fonds nécessaires que le projet peut continuer. Le projet est principalement financé par quatre sourcesMaryknoll (la mission catholique basée aux États-Unis à laquelle appartient Liz), la Oak Foundation, le Women's Dignity Project (WDP) ainsi que les donations de la propre famille de Liz et de ses amis. En outre, la Fondation pour la médecine et la recherche en Afrique (AMREF) verse 100 dollars (financé par I'Ambassade des Pays-Bas) pour chaque

Étant donné la grave pénurie en médecins dont souffre la Tanzanie, le nombre d'obstétriciens-gynécologues et chirurgiens pouvant pratiquer des réparations chirurgicales de fistule est extrêmement restreint. Il faut compter sur les médecins étrangers en visite pour fournir ce service dont le pays a tant besoin. Le Docteur Kelly pratique encore environ $50 \%$ des réparations chirurgicales de fistule chaque année et le Docteur Raassen près de $10 \%$. Le personnel local assiste souvent aux interventions chirurgicales délicates pour se former, en plus de leurs tâches quotidiennes visant à donner le maximum de chances aux patientes pour qu'elles puissent se rétablir complètement.

Toutefois, compter sur les chirurgiens en visite complique le fonctionnement du service : les chirurgiens tanzaniens préférant le plus souvent laisser les cas les plus compliqués aux chirurgiens en visite, certaines patientes restent en attente plusieurs mois dans le service. Pour tirer le maximum du séjour des chirurgiens en visite, le personnel doit se mobiliser plusieurs semaines à l'avance, passer des annonces à la radio et envoyer des courriels aux camps de réfugiés et aux hôpitaux de district. Avant l'arrivée du médecin en visite le personnel du bloc 
opératoire, les anesthésistes et les infirmières revoient leurs listes et commandent les médicaments, les agrafes, les sondes et autres fournitures nécessaires pour que tout soit disponible lorsque I'intervenant en aura besoin.

\section{La motivation du personnel soignant}

Le personnel local ne travaille pas dans le service à l'origine pour l'appât du gain. En effet, les salaires dans les hôpitaux d'État sont si bas qu'une grande partie du personnel a un deuxième travail dans le secteur privé. Les réparations de fistule sont peu lucratives car les patientes sont généralement pauvres et leur opération longue, ce qui au début du projet a posé de sérieux problèmes : 1) Il était difficile de trouver du personnel, notamment des médecins, et de le garder. 2) Les chirurgiens souvent passaient le tour des patientes souffrant de fistule pour opérer à leur place des cas plus urgents ou rapportant plus. Pour remédier à cette situation on a donc décidé que les réparations chirurgicales de fistule se pratiqueraient après les heures de travail normales et que le personnel serait payé en heures supplémentaires à raison de 12 dollars par jour pour les médecins et huit pour les infirmières.

Le fait de payer des heures supplémentaires au personnel pratiquant les réparations chirurgicales de fistules a, au début, créé des tensions dans I'hôpital. Le personnel des autres départements se demandait ce que les patientes souffrant de fistule avaient de si particulier pour que leur traitement mérite d'être payé en heures supplémentaires. Tous ceux ayant à faire, de près ou de loin, avec les patientes souffrant de fistules, du personnel de la cuisine aux pharmaciens, ne comprenaient pas pourquoi ils ne touchaient pas un supplément eux aussi même s'ils ne faisaient pas d'heures supplémentaires.
Mais d'après Liz Mach, ce système d'heures supplémentaires a permis de constituer une équipe solide et, couplé avec la stricte réglementation de I'hôpital, de diminuer la corruption qui est toujours un problème là où les gens doivent se battre pour leur survie quotidienne. Pour lutter contre la trop grande mobilité des médecins, une tendance généralisée en Tanzanie, et les retenir, le Docteur Majinge a décidé d'autoriser tous les médecins de l'hôpital à avoir leur clientèle privée. Cela a aussi permis d'augmenter le nombre de membres du personnel hospitalier percevant un revenu supplémentaire et ainsi d'atténuer certains mécontentements suscité par le projet.

\section{La qualité des soins infirmiers}

Des soins infirmiers de qualité sont indispensables pour assurer la réparation des fistules. Le projet insiste énormément sur le développement des compétences du personnel infirmier, ce qui pose d'énormes difficultés compte tenu de I'augmentation exponentielle du nombre de patientes.

La formation des nouveaux membres de l'équipe est extrêmement importante (quatre nouvelles personnes ont été recrutées avec l'ouverture de la nouvelle section). Le comité du projet encourage des spécialistes à venir animer sur place des ateliers au cours desquels les participants les voient pratiquer. Le Comité organise également d'autres types de formation qui vont d'une approche informelle (formation sur le tas des nouvelles infirmières par une infirmière expérimentée) à des séminaires bien structurés d'un ou deux jours. Un séminaire obligatoire a eu lieu en 2001 pour toutes les infirmières et assistantes infirmières du service. Les résultats ont été très positifs. Les formateurs ont présenté deux scénarios dans lesquels des 


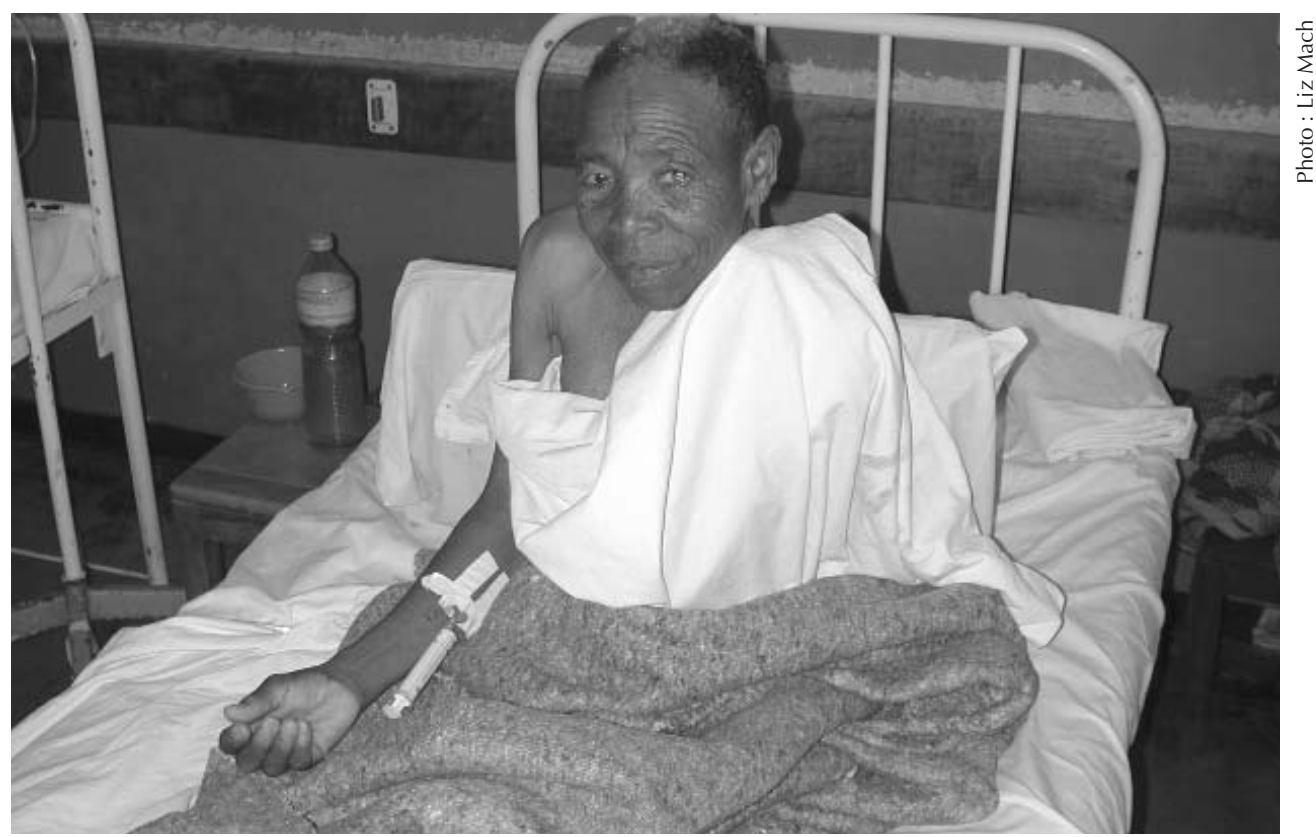

L'infirmière chef du projet dit à ses infirmières, "Mettez-vous à la place des patientes et essayez d'imaginer ce qu'elles ressentent ».

patientes recevaient des soins pré- et post-opératoires et ont demandé aux participantes d'identifier les erreurs sciemment commises. Un autre scénario était axé sur la réticence qu'ont parfois les infirmières à discuter avec les médecins en visite des soins à fournir aux patientes ou à leur poser des questions. Ces activités ont suscité de vifs débats (et des rires) et ont permis de motiver le groupe et d'augmenter sa cohésion.

Yasinta Mkama (infirmière chef du service des fistules) contrôle étroitement ses infirmières et insiste en permanence sur l'atmosphère que le projet cherche à créer pour les patientes. "Certaines infirmières, raconte-elle, lorsqu'elles arrivent dans le service parlent mal aux patientes et ne les traitent pas bien. Alors je leur dis "Qu'est-ce que vous ressentiriez si c'était votre mère ou votre sœur qui avait une fistule et que quelqu'un la traite comme ça? » ou encore "Ça pourrait facilement être vous qui demain ayez une fistule; mettez-vous toujours à la place des patientes et essayez d'imaginer ce qu'elles ressentent. » Si une infirmière ne se sent pas à l'aise dans le service elle peut demander un transfert mais l'équipe du projet s'efforce de garder les infirmières qui ont été formées. L'année dernière seulement deux infirmières ont quitté le service.

\section{Au-delà de Bugando: Un partenariat national de lutte contre la fistule obstétrique}

La réparation chirurgicale est un aspect essentiel de la lutte contre cette lésion dévastatrice qu'est la fistule, mais une approche globale est indispensable : sensibilisation aux possibilités de réparation; identification des femmes ayant besoin $d^{\prime}$ une opération; mise au point de plans stratégiques pour faciliter et gérer les soins; mobilisation des ressources nationales et internationales; et 
surtout, réduction de l'incidence des fistules obstétriques en élargissant l'accès des femmes et des jeunes filles pauvres aux soins obstétriques d'urgence et en insistant sur les droits des femmes et leur santé. La Tanzanie dispose d'un réseau extrêmement solide regroupant ONG, hôpitaux, organismes gouvernementaux et organisations internationales travaillant sur ces questions. Les membres de ce réseau échangent leurs expériences, des informations et des conseils, fournissent une assistance financière et diffusent les histoires particulièrement émouvantes de certaines patientes. Les principaux acteurs sont, outre l'équipe de Bugando, les suivants :

Le Gouvernement joue un rôle essentiel dans l'effort national de lutte contre la fistule obstétrique. Le Docteur Zachary Berege qui, lorsqu'il était directeur de I'hôpital, a réellement aidé à mettre le projet en place, a quitté Bugando en 2000 pour devenir Directeur des services hospitaliers du Ministère de la santé. Depuis il a largement contribué à sensibiliser l'opinion à la fistule obstétrique, à obtenir des fonds sur le budget national pour son traitement et a réellement fait avancer la lutte contre cette lésion dévastatrice en mettant en place un système national d'orientation des malades vers les centres de traitement appropriés.

Le Women's Dignity Project (WDP) a été fondé par Maggie Bangser en 2000 avec l'objectif de traiter la fistule obstétrique dans le contexte plus large de l'impact primordial des politiques de la santé et d'allocation des ressources sur l'état de santé des pauvres. WDP emploie 15 personnes et travaille dans quatre domaines, à savoir : 1) la recherche au niveau des communautés-analyse des liens entre fistule et vulnérabilité sociale et planification (facteurs socioéconomiques pouvant augmenter le risque de fistule obstétrique); 2) le développement des capacités organisationnelles pour le traitement des fistules obstétriques et I'accès équitable des deux sexes à la santé; 3) I'analyse politique et critique; 4) la mobilisation des ressources pour la réparation des fistules obstétriques.

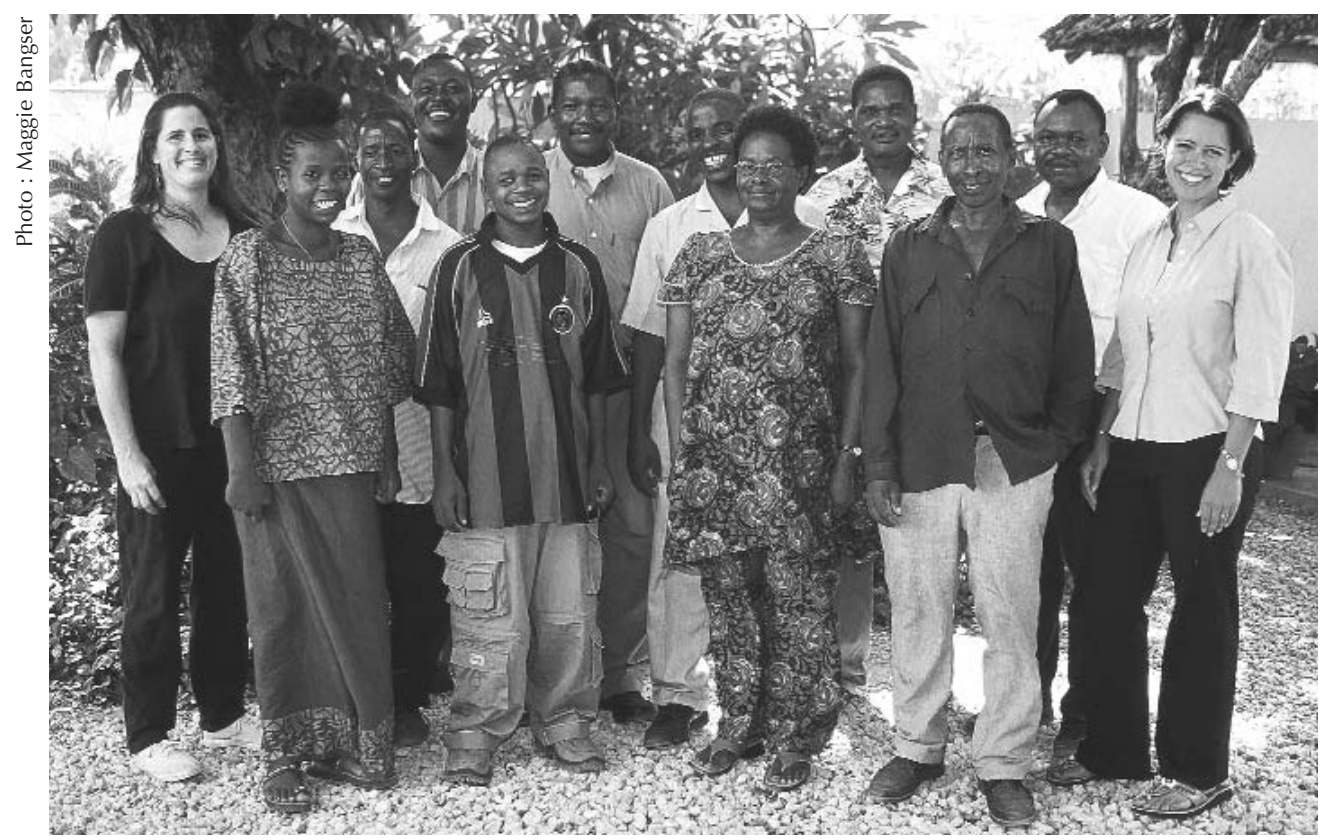

Le personnel du Women's Dignity Project 
L'Association des sages-femmes tanzaniennes-Tanzania Midwives Association (TAMA) - cherche à éduquer les chefs de village, les patientes et les membres des communautés sur tout un ensemble de questions touchant à la santé reproductive et à la santé des mères et des nourrissons, dont les problèmes de fistule obstétrique. L'Association travaille

Une ONG travaillant au niveau

des villages sur le problème des mutilations génitales féminines a récemment identifié sept femmes souffrant de fistule (y compris une jeune fille de 17 ans qui avait été battue pendant son accouchement qui durait trop longtemps pour accélérer la naissance). L'organisation

a pris contact avec le Women's Dignity Project qui a pris des dispositions pour le transport et le traitement de ces femmes dans un hôpital de Dar-es-Salaam.

en étroite collaboration avec le Projet de Bugando et a même une branche à Bugando. L'une de ses responsables, Esther Mashauri, a été I'un des membres du comité originel du projet.

Le Gouvernement, I'hôpital de Bugando et la société civile collaborent pour gagner la bataille contre la fistule obstétrique. Les mesures prises incluent notamment :

\section{La stratégie pour élargir la demande de traitement}

Une femme ou une jeune fille souffrant d'une fistule obstétrique, en général, ne comprend pas ce qui lui arrive et ignore qu'il existe un traitement efficace. C'est pourquoi le réseau cherche, par de nombreux moyens, à éduquer la population en expliquant ce qu'est la fistule obstétrique et où s'adresser pour un traitement. Les membres de I'Association des sages-femmes tanzaniennes actives dans la région du Lac montrent des vidéos dans les hôpitaux, les dispensaires et les centres de santé, passent des annonces à la radio, participent aux campagnes d'affichage sur la fistule obstétrique et les activités du Projet de Bugando. Le personnel de Bugando participe également à des entretiens radiophoniques en anglais et en swahili et une chaîne télévisée a consacré un programme de 30 minutes au projet.

Début février 2004, le WDP a lancé dans les médias une campagne d'information intensive sur la fistule pour accompagner la publication de son fascicule Faces of dignity: Seven Stories of Girls and Women with Fistula (Retrouver sa dignité : Histoires de sept femmes et jeunes filles souffrant d'une fistule). Le Ministre de la santé, Mme Anna Abdallah, a inauguré officiellement cette campagne par un discours dans lequel elle expliquait sa motivation personnelle et sa volonté d'aider les femmes souffrant de fistule : Sa propre grand-tante a souffert d'une fistule pendant des années, ce qui lui provoquait difficultés et manque de bien-être. Faces of Dignity et sa version swahili Sura za utu ont été diffusées dans trois journaux, touchant globalement 60000 lecteurs.

La collaboration étroite avec les ONG est très payante. Une ONG travaillant au niveau des villages sur le problème des mutilations génitales féminines a récemment identifié sept femmes souffrant de fistule (y compris une jeune fille de 17 ans qui avait été battue pendant son accouchement qui durait trop longtemps pour accélérer la naissance). L'organisation a pris contact avec le Women's Dignity Project qui a pris des dispositions pour le transport et 
le traitement de ces femmes dans un hôpital de Dar-es-Salaam.

Le nombre de femmes souffrant de fistule et qui ont besoin d'un traitement est énorme parce qu'il faut rattraper un retard de plusieurs dizaines d'années. Récemment le WDP, au cours d'une recherche sur le terrain dans quelques villages, a identifié en seulement deux semaines 16 femmes souffrant de fistule. Bon nombre d'entre elles étaient des femmes âgées, dont deux ayant vécu avec leur lésion pendant plus de 35 ans. Ces femmes, les unes après les autres, ont rejoint " la caravane » qui s'est acheminée avec les chercheurs vers Bugando pour être soignées.

\section{Le renforcement des capacités nationales}

La mise en place d'un système d'orientation des patientes vers les centres de traitement est un élément crucial du programme tanzanien de lutte contre la fistule obstétrique. En 2001, le Ministère de la santé et le WDP ont, ensemble, établi et envoyé un questionnaire succinct à tous les hôpitaux (de district, régionaux et de mission) du pays pour recenser les régions et les hôpitaux dans lesquels un traitement de la fistule obstétrique était possible et examiner les obstacles à l'accès des femmes à ces services. (Voir encadré sur les principaux résultats de l'enquête sur les fistules en Tanzanie).

Les résultats de cette enquête ont été utilisés pour préparer la mise en place $d^{\prime}$ un système national d'orientation. Des représentants du Ministère de la santé, du WDP, de huit hôpitaux, des organisations de donateurs et autres partenaires se sont réunis en octobre 2002 pour étudier la mise en place $d^{\prime}$ un système d'orientation efficace qui permettra de renforcer les soins dispensés. Ils ont adopté un système à trois niveaux. Le premier niveau rassemble tous les hôpitaux dispensant des soins d'obstétrique

\section{Conclusions de l'enquête sur la fistule obstétrique en Tanzanie}

Le questionnaire a été envoyé à 172 hôpitaux. Cent trente-huit ( 80 \%) y ont répondu, ce qui prouve le grand intérêt que suscite la question. L'enquête a donné les résultats suivants :

1. Cinquante hôpitaux ont, au total, pratiqué 712 réparations chirurgicales en 2000-01. Le nombre de réparations pratiquées par hôpital va de une à 172 .

2. Les cinq principaux hôpitaux pratiquant les réparations sont situés sur le pourtour du pays, si bien que le traitement reste en grande partie inaccessible aux gens de l'intérieur.

3. Seulement 12 médecins tanzaniens ont été formés pour traiter les fistules obstétriques. Les hôpitaux qui pratiquent des réparations chirurgicales dépendent, pour ces opérations, de chirurgiens étrangers en visite.

4. Le coût du traitement et du transport pour y accéder sont des obstacles infranchissables pour beaucoup de femmes. Bien que de nombreux hôpitaux aient un système de partage des coûts, les patientes doivent payer en moyenne 26 dollars pour leur traitement. De nombreuses patientes doivent parcourir plus de 500 kilomètres pour parvenir à un des grands centres pratiquant les réparations.

5. Dix-neuf hôpitaux pratiquant des réparations chirurgicales manquent du matériel chirurgical requis et ont des équipements désuets. 
avec un personnel formé pour la prévention, le diagnostic et le traitement précoce des fistules par installation d'une sonde. Le deuxième niveau regroupe les huit hôpitaux situés dans les zones où aucun soin n'existe mais dont la capacité clinique peut être renforcée pour pratiquer des réparations simples et identifier les cas les plus délicats pour les envoyer aux centres du troisième niveau. Le troisième niveau comprend les cinq hôpitaux dans lesquels les médecins et le personnel infirmier peuvent traiter un grand nombre de patientes souffrant de fistules, notamment les cas difficiles. Les médecins des centres du troisième niveau assureront la formation des médecins des centres du deuxième niveau. ${ }^{3}$

Le système d'orientation a été modifié en tenant compte des réponses des prestataires de soins et des femmes souffrant de fistule pour permettre la formation d'un plus grand nombre de médecins des hôpitaux du deuxième niveau. Pour lever en partie les nombreux obstacles auxquels sont confrontées les femmes qui doivent voyager de longues distances pour atteindre un centre de soins, il a été décidé de multiplier les centres pouvant pratiquer des réparations simples et d'augmenter leur capacité, tout en veillant à mieux les répartir.

\section{Le renforcement de la base de ressources}

Le réseau cherche à trouver de nouvelles sources de financement, internationales et nationales, pour la lutte contre la fistule obstétrique, notamment pour les réparations chirurgicales. L'appui et I'engagement du Ministre de la santé et du Secrétaire permanent pour la santé, Mme Mariam Mwaffisi, jouent un rôle déterminant pour la sensibilisation au problème et l'augmentation des ressources. Grâce en grande partie à leurs efforts, 50000 dollars ont été alloués sur le budget du Ministère de la santé expressément aux réparations de fistules.

Le réseau cherche aussi à augmenter les ressources du secteur de la santé en général. Le gouvernement et les autres acteurs impliqués ont prouvé leur engagement en invitant le WDP à siéger aux deux principaux comités chargés de trouver de nouvelles ressources pour le secteur de la santé et d'aider à contrôler les stratégies de réduction de la pauvreté. Le WDP, en participant à ces comités, peut trouver de nouveaux partenaires et influencer les décisions ayant un impact direct sur la vie des personnes démunies. En outre, grâce au réseau, la lutte contre la fistule obstétrique est maintenant inscrite au programme général de santé reproductive. Le réseau collabore également avec les organisations internationales concernées.

\section{La stratégie nationale de prévention}

La prévention vise deux objectifs étroitement liés : la modification des attitudes de la famille et de la communauté qui n'accordent aucune valeur à la santé des femmes et des jeunes filles et l'élargissement de l'accès des femmes aux soins prénatals et obstétricaux d'urgence, tout en améliorant leur qualité.

En 1997, en collaboration avec d'autres ONG basées à Mwanza et

\footnotetext{
${ }^{3}$ Le personnel de Bugando, dans le cadre du Programme Specialist Outreach de I'AMREF, se rend dans les hôpitaux isolés. Les chirurgiens pratiquent des réparations chirurgicales, font des exposés, apprennent au personnel médical local à participer aux opérations. Ils cherchent également à inciter les chirurgiens à se former. Elizabeth Bushiri, infirmière en chef du bloc opératoire de Bugando, anime fréquemment des séminaires d'une journée destiné aux infirmières et assistantes infirmières des blocs opératoires des environs.
} 
avec le Centre Kuleana de défense des droits de l'enfant, le Projet fistule a produit un fascicule intitulé en swahili Haji Zangu, Je? et en anglais What About My Rights? (Et mes droits?). Cette brochure bilingue swahili/anglais raconte I'histoire d'Eliza, une adolescente contrainte d'avoir une relation sexuelle avec un homme plus âgé qui lui donne de l'argent pour payer l'école. Elle tombe enceinte et après l'accouchement souffre d'une fistule. La conclusion de l'histoire, illustrée de façon très vivante, est que "Tout le monde, y compris les femmes et les petites filles, a droit à l'éducation, à des soins de santé de qualité, à être traité sans violence, à une alimentation nutritive, au repos et à la relaxation, à décider, à être respecté, à être reconnu à leur juste valeur et aimé ». Ce fascicule a été distribué à 50000 exemplaires dans les écoles et les centres de santé ainsi qu'aux groupes communautaires et aux responsables religieux.

L'Association des sages-femmes tanzaniennes cherche à améliorer la situation en matière d'obstétrique en encourageant les soins cliniques de qualité, en luttant pour améliorer le statut social des filles, en particulier en repoussant l'âge de la première grossesse. Pour que les sages-femmes évoluent avec leur profession, I'Association organise des ateliers sur les soins anténatals et obstétricaux (y compris comment reconnaître les grossesses à haut risque et dispenser les soins requis en cas d'accouchement difficile). L'Association a également eu des discussions positives avec les chefs religieux sur les dangers des mariages précoces. Dans le cadre d'un séminaire de formation organisé par l'Association, les travailleurs de santé locaux se sont impliqués avec les membres de leur communauté dans un concours d'affiches sur le droit des femmes et des filles aux soins de santé. Une affiche dépeignait une jeune fille refusant une offre de mariage parce qu'elle n'avait pas encore fini l'école, une autre des hommes transportant de toute urgence une femme enceinte nécessitant des soins dans un hôpital. L'équipe du Projet de Bugando a utilisé certaines de ces affiches pour un calendrier en 1999. Elle a aussi engagé un artiste local pour reproduire ces images en fresques murales qui ont été montrées dans toutes les communautés participantes. L'équipe espère que ces fresques murales exposées dans des lieux publics comme les marchés et les hôpitaux de district amèneront le public à s'interroger sur les raisons sous-jacentes à la mauvaise santé reproductive des femmes.

Le WDP, en collaboration avec quatre partenaires locaux et EngenderHealth a lancé une recherche sur les causes de vulnérabilité aux fistules obstétriques. L'objectif est, grâce à des entretiens, des débats de groupes thématiques, des méthodes de recherche participatives

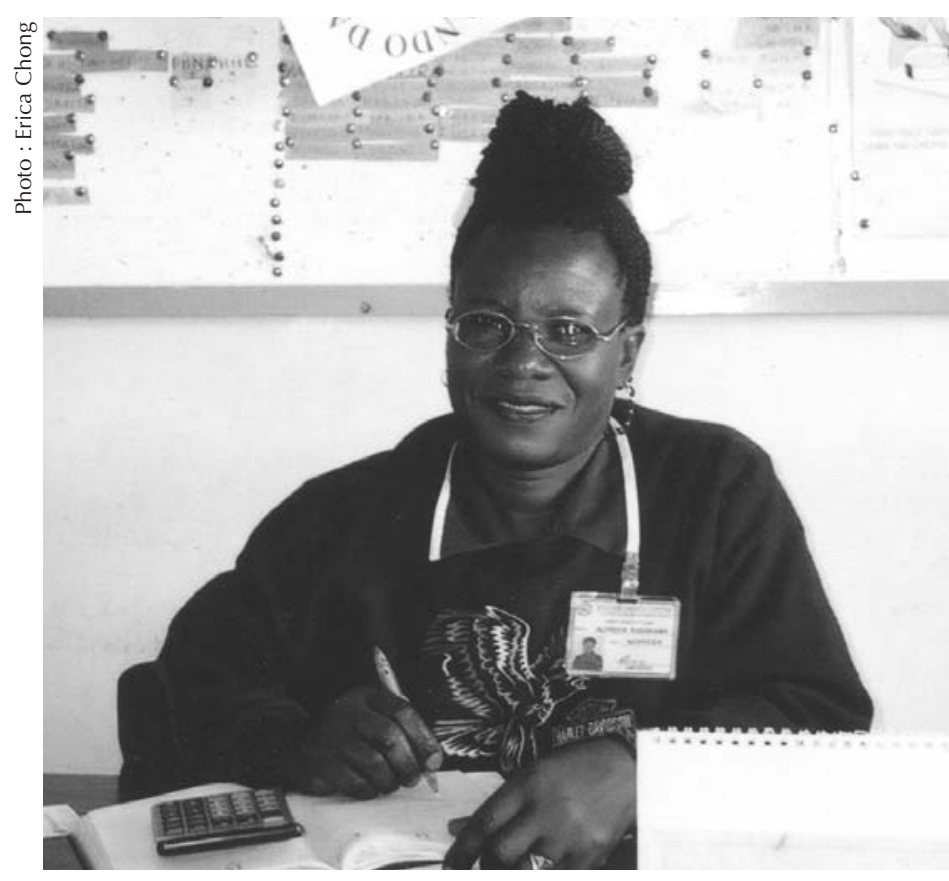

Mme Alfreda Kabakama est la présidente de la branche du Centre médical de Bugando de l'Association des sages-femmes tanzaniennes (TAMA). L'association cherche à décourager le mariage des enfants et à promouvoir les droits des femmes et des jeunes filles. 
faisant appel aux jeunes filles et aux femmes, aux membres de la famille, aux membres de la communauté et aux prestataires de soins, d'évaluer les facteurs socioéconomiques qui rendent les

\section{Le coût d'une intervention}

\section{chirurgicale est de très loin inférieur}

à la perte enregistrée lorsqu'une

femme est marginalisée socialement

pour le reste de sa vie. En outre, le

lancement d'un programme de lutte

contre la fistule est relativement

peu coûteux.

\section{Les enseignements}

De nombreux prestataires de soins et décideurs, comprenant que les fistules ne sont pas seulement dues à l'absence de soins d'obstétrique d'urgence mais également à la pauvreté et à l'absence d'équité entre les sexes, se sont montrés découragés par l'immensité de la tâche pour faire de la réparation des fistules obstétriques un soin de santé prioritaire. Prévenir la formation des fistules semble même être une entreprise encore plus gigantesque. Mais les résultats obtenus par le réseau de lutte contre la fistule obstétrique en Tanzanie prouvent que la collaboration entre les hôpitaux, le gouvernement et les communautés peut déboucher sur un effort multidimensionnel d'interventions au niveau économique, social et technique. Le réseau offre un modèle prometteur de lutte contre cette lésion dévastatrice partout dans le monde.

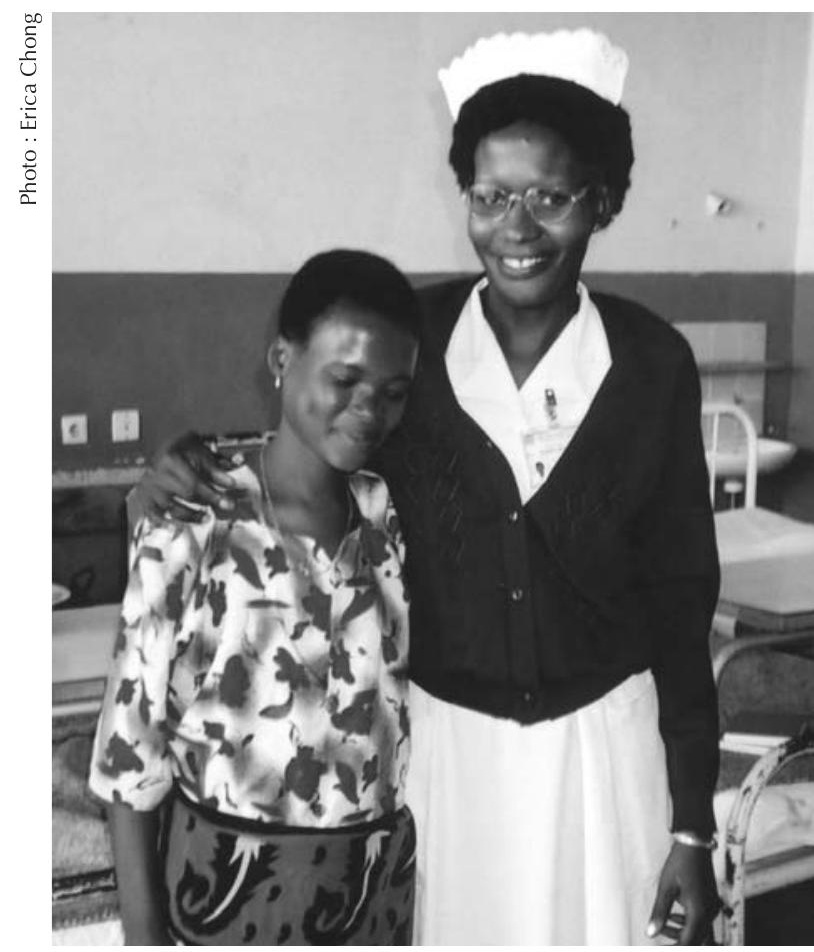

La grande majorité des fistules peuvent être réparées avec succès si l'opération est faite dans des conditions appropriées.

jeunes filles et les femmes vulnérables, déterminent les conditions dans lesquelles elles accouchent, la période puerpérale ainsi que leur accès aux soins. La recherche a mis à jour des facteurs invisibles comme les coûts d'opportunité occasionnés en quittant le domicile, les modes de fonctionnement qui font que les pauvres sont les derniers à recevoir des traitements, I'absence de systèmes de transport communautaires d'urgence et enfin l'ignorance, chez les chefs de famille (la plupart du temps les maris ou les bellesmères), des dangers des mariages et des grossesses trop précoces et de la nécessité de soins médicaux d'urgence en cas d'accouchement difficile. Les résultats seront utilisés pour trouver des moyens locaux de prévenir les fistules et de les traiter en coopération avec les communautés et les prestataires de soins de santé et de vaincre ce fléau national. Une deuxième étude du WDP porte sur les femmes pauvres et les soins de santé - prix des soins, obstacles à l'accès aux soins et qualité générale des soins dispensés. 
Pour lutter efficacement contre la fistule obstétrique une approche multidimensionnelle est nécessaire. Même les programmes qui se limitent au départ à I'aspect clinique de la réparation chirurgicale (plutôt que la prévention) se trouvent rapidement confrontés aux problèmes socioéconomiques (pénurie de chirurgiens, réactions négatives du personnel de I'hôpital et du service de chirurgie, nécessité de conseiller et d'épauler patiemment les patientes, manque de sensibilisation du public, mépris pour les besoins des femmes, problèmes de transport, problèmes de réintégration des patientes et de financement des soins).

Bien que la réparation des fistules ne soit pas une réussite à $100 \%$, la grande majorité des lésions peuvent être réparées avec succès ou au moins la condition de la patiente est radicalement améliorée si la réparation est faite dans les conditions appropriées. Et la différence positive qu'apporte à la vie d'une femme une réparation réussie est énorme.

Les décideurs, les chirurgiens et les donateurs qui considéraient la fistule obstétrique comme un problème écrasant et insoluble sont souvent inspirés par les histoires de patientes. Ces exemples positifs leur donnent le courage de s'attaquer au problème et de croire à la réussite.

La réparation de la fistule obstétrique est aussi un bon investissement économique. Une intervention revient à environ 250 dollars, ce qui est de très loin inférieur à la perte enregistrée lorsqu'une femme est marginalisée socialement pour le reste de sa vie. Enfin, le lancement d'un programme de lutte contre la fistule est relativement peu coûteux.

L'incidence de la fistule est le reflet des problèmes auxquels les femmes pauvres sont généralement confrontées : les efforts déployés pour réduire la fréquence de cette lésion peuvent aussi aider à sensibiliser à l'oppression des femmes et à leur droit aux soins de santé.

\section{Références}

Bangser, Maggie et al. 1999. «A comprehensive approach to vesico-vaginal fistula: A project in Mwanza, Tanzania », dans Safe Motherhood Initiatives: Critical Issues. Marge Berer et T.K. Sundari Ravindran (éds.). Oxford: Blackwell Science, pages 157-165.

Banque mondiale. 2003. Country Data: Tanzania at a Glance. <http://www. worldbank.org/data/countrydata/countrydata.html>. Accedé le 11 juillet 2003.

Mpangile, Gottlieb S. et al. 1993. " Factors associated with induced abortion in public hospitals in Dar es Salaam, Tanzania », Reproductive Health Matters 2, pages 21-31.

National Bureau of Statistics of Tanzania (NBS) et Macro International (MI). 2000. Tanzania Reproductive and Child Health Survey 1999. Dar-es-Salaam et Calverton MD : NBS et MI.

Population Reference Bureau (PRB). 2000. The World's Youth 2000. Washington DC: PRB.

2001. Youth in Sub-Saharan Africa: A Chartbook on Sexual Experience and Reproductive Health. Washington DC : PRB.

2002. 2002 World Population Data Sheet. Washington DC : PRB.

The POLICY Project (TPP). Maternal and Neonatal Program Effort Index: Tanzania. $<$ http://www.policyproject.com/pubs/ MNPI/Tanzania_MNPI.pdf $>$. Accedé le 11 juillet 2003.

Programme des Nations unies pour le développement (PNUD). 2003. Rapport mondial sur le développement humain 2003. Paris : ECONOMICA.

UNFPA (Fonds des Nations unies pour la population). 2003. Deuxième réunion du Groupe de travail sur la prévention et le traitement de la fistule obstétrique. New York : UNFPA.

Women's Dignity Project (WDP). 2002. Tanzania Fistula Survey 2001. Dar-esSalaam : WDP. 


\section{À propos des auteurs}

Thoraya Ahmed Obaid est Directrice exécutive du Fonds des Nations unies pour la population (UNFPA), la plus importante source internationale, dans le monde entier, de financement des programmes de population. L'UNFPA mène une campagne mondiale pour éliminer les fistules.

Erica Chong est assistante à la Division des programmes internationaux du Population Council à New York.

\section{Personnel de production}

\section{Directrice de la rédaction :}

Debbie Rogow

Assistante de recherche

et de rédaction : Michelle Skaer

Graphiste : Mike Vosika

Traducteur : Multi-Language Services, Inc.

\section{Groupe consultatif}

Delia Barcelona

Gary Barker

Carmen Barroso

Judith Bruce

Batya Elul

Susana Galdos

Kirrin Gill

Françoise Girard

Nicole Haberland

Katherine Kurz
Laura Laski

Ann Leonard

Ann McCauley

Liz McGrory

Manisha Mehta

Suellen Miller

Saumya Ramarao

Julie Reich

Ann Starrs

Cynthia Steele

\section{Remerciments}

Erica Chong tient à remercier le personnel dévoué du Centre médical de Bugando, en particulier Elisabeth Mach et Yasinda Mkama, pour les précieuses informations qu'elles ont fournies et pour leur aide dans la collecte des données. Elle est également très reconnaissante aux femmes et jeunes filles qui ont partagé avec elle leurs observations sur leur expérience de la fistule.

Nous serions heureux de recevoir vos commentaires sur Quality/Calidad/Qualité. Si vous souhaitez être inclus dans notre fichier d'adresses, veuillez nous envoyer un courrier électronique à l'adresse suivante : qcq@popcouncil.org. La plupart des éditions précédentes sont disponibles en ligne à l'adresse suivante : <www.popcouncil.org/publications/qcq/ default.htm>. Les ouvrages suivants sont également disponibles; vous pouvez commander une ou plusieurs copies par courrier électronique :

Celebrating Mother and Child on the Fortieth Day: The Sfax Tunisia Postpartum Program (en anglais seulement), $\mathrm{n}^{\circ}$ 1, 1989.

Man/Hombre/Homme: Meeting Male Reproductive Health Care Needs in Latin America (en anglais et espagnol), $\mathrm{n}^{\circ} 2,1990$.

The Bangladesh Women's Health Coalition (en anglais seulement), nº 3, 1991.

Gente Joven/Young People: A Dialogue on Sexuality with Adolescents in Mexico (en anglais et espagnol), $\mathrm{n}^{\circ}$ 5, 1993.

The Coletivo: A Feminist Sexuality and Health Collective in Brazil (en anglais, portugais et espagnol), n 6, 1995.

Doing More with Less: The Marie Stopes Clinics of Sierra Leone (en anglais seulement), $\mathrm{n}^{\circ}$ 7, 1995.

Introducing Sexuality within Family Planning: Three Positive Experiences from Latin America and the Caribbean (en anglais et espagnol), $\mathrm{n}^{\circ}$ 8, 1997.
Using COPE to Improve Quality of Care: The Experience of the Family Planning Association of Kenya (en anglais et espagnol), $n^{\circ} 9$, 1998.

Alone You Are Nobody, Together We Float: The Manuela Ramos Movement (en anglais et espagnol), $\mathrm{n}^{\circ} 10,2000$.

From Patna to Paris: Providing Safe and Humane Abortion (en anglais seulement), $\mathrm{n}^{\circ}$ 11, 2001.

Universal Sexuality Education in Mongolia: Educating Today to Protect Tomorrow (en anglais seulement), $\mathrm{n}^{\circ} 12,2002$.

What about Us? Bringing Infertility into Reproductive Health Care (en anglais seulement), no 13, 2002.

"My Father Didn't Think This Way": Nigerian Boys Contemplate Gender Equality (en anglais et français), $n^{\circ}$ 14, 2003.

Healing Wounds, Instilling Hope: The Tanzanian Partnership Against Obstetric Fistula (en anglais), $n^{\circ}$ 15, 2004. 
Q/C/Q No 16 français

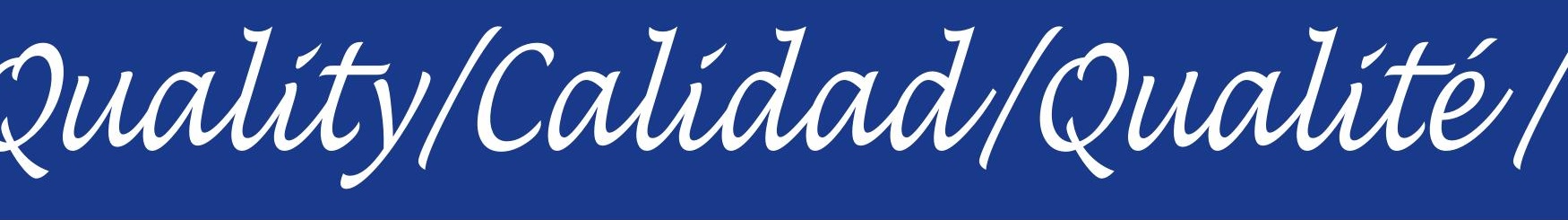

